\title{
Kinetics of photoinduced ordering in azo-dye films: Two-state and diffusion models
}

\author{
Alexei D. Kiselev, ${ }^{1,2, *}$ Vladimir G. Chigrinov, ${ }^{1, \dagger}$ and Hoi-Sing Kwok ${ }^{1, \ddagger}$ \\ ${ }^{1}$ Hong Kong University of Science and Technology, Clear Water Bay, Kowloon, Hong Kong \\ ${ }^{2}$ Institute of Physics, Institute of Physics of National Academy of Sciences of Ukraine, Prospekt Nauki 46, 03028 Kÿ̈v, Ukraine
}

(Received 5 April 2009; published 21 July 2009)

\begin{abstract}
We theoretically study the kinetics of photoinduced ordering in azo-dye photoaligning layers and present the results of modeling performed using two different phenomenological approaches. A phenomenological twostate model is deduced from the master equation for the one-particle distribution functions of an ensemble of two-level molecular systems by specifying the angular redistribution probabilities and by expressing the order parameter correlation functions in terms of the order parameter tensor. Using an alternative approach that describes light-induced reorientation of azo-dye molecules in terms of a rotational Brownian motion, we formulate the two-dimensional diffusion model as the free energy Fokker-Planck equation simplified for the limiting regime of purely in-plane reorientation. The models are employed to interpret the irradiation time dependence of the absorption order parameters defined in terms of the principal extinction (absorption) coefficients. Using the exact solution to the light transmission problem for a biaxially anisotropic absorbing layer, these coefficients are extracted from the absorbance-vs-incidence angle curves measured at different irradiation doses for the probe light linearly polarized parallel and perpendicular to the plane of incidence. It is found that, in the azo-dye films, the transient photoinduced structures are biaxially anisotropic whereas the photosteady and the initial states are uniaxial.
\end{abstract}

DOI: 10.1103/PhysRevE.80.011706

PACS number(s): $61.30 . \mathrm{Gd}, 42.70 . \mathrm{Gi}, 82.50 . \mathrm{Hp}$

\section{INTRODUCTION}

It has long been known that some photosensitive materials such as compounds containing azobenzene and its derivatives may become dichroic and birefringent under the action of light. This phenomenon-the so-called effect of photoinduced optical anisotropy (POA) - has a long history dating back nine decades to the paper by Weigert [1].

Over the past few decades the Weigert effect (POA) has been attracted much attention because of its importance in the development of tools dealing with the light-controlled anisotropy. For example, the materials that exhibit POA are very promising for use in many photonic applications [2-5].

It is also well known that one of the key procedures in the fabrication of liquid crystal electro-optic devices is the production of substrates with anisotropic anchoring properties. The traditional method widely used to align liquid crystal display cells involves mechanical rubbing of aligning layers and has a number of the well-known difficulties [6]. The photoalignment technique suggested in Refs. [7-9] is an alternative method that avoids the drawbacks of the mechanical surface treatment by using linearly polarized ultraviolet (UV) light to induce anisotropy of the angular distribution of molecules in a photosensitive film $[10,11]$. Thus the phenomenon of POA-the so-called Weigert effect-is at the heart of the photoalignment method.

Light induced ordering in photosensitive materials, though not being understood very well, can generally occur in a variety of photochemically induced processes. These typically may involve such transformations as photoisomer-

\footnotetext{
*kiselev@iop.kiev.ua

†eechigr@ust.hk

†.eekwok@ust.hk
}

ization, cross-linking, photodimerization, and photodecomposition (a recent review can be found in Refs. [11,12]).

So, the mechanism underlying POA and its properties cannot be universal. Rather they crucially depend on the material in question and on a number of additional factors such as irradiation conditions, surface interactions, etc. In particular, these factors combined with the action of light may result in different regimes of the photoinduced ordering kinetics leading to the formation of various photoinduced orientational structures (uniaxial, biaxial, and splayed).

POA was initially studied in viscous solutions of azo-dyes [13] and in azo-dye-polymer blends [14], where the anisotropy was found to be rather unstable. This is the case where the photoinduced anisotropy disappears after switching off the irradiation [13-18]. By contrast to this case, POA can be long-term stable.

The stable POA was observed in polymers containing chemically linked azochromophores (azopolymers) [2]. It turned out that stable anisotropy can be induced in both amorphous and liquid crystalline azopolymers [2,3,19-23].

The photoalignment has also been studied in a number of similar polymer systems including dye doped polymer layers $[7,24]$, cinnamate polymer derivatives $[8,9,25,26]$, and side chain azopolymers $[19,20,22,27]$. In addition, the films containing photochemically stable azo-dye structures (azobenzene sulfuric dyes) were recently investigated as new photoaligning materials for nematic liquid crystal cells [28-30].

In Ref. [29], it was found that, owing to high degree of the photoinduced ordering, these films used as aligning substrates are characterized by the anchoring energy strengths comparable to the rubbed polyimide films. For these materials, the voltage holding ratio and thermal stability of the alignment turned out to be high. The azo-dye films are thus promising materials for applications in liquid crystal devices.

According to Ref. [30], the anchoring characteristics of the azo-dye films such as the polar and azimuthal anchoring 
energies are strongly influenced by the photoinduced ordering. In this paper the kinetics of such ordering will be of our primary interest. More specifically, we deal with theoretical approaches and related phenomenological models describing how the amount of the photoinduced anisotropy characterized by absorption dichroism evolves in time upon illumination and after switching it off.

There are a number of models [17,23,31-35] formulated for azocompounds exhibiting POA driven by the trans-cis photoisomerization. In these models, a sample is treated as an ensemble of the stable trans isomers characterized by elongated rodlike molecular conformation and the bent bananalike shaped cis isomers. The mechanism of photoisomerization implies that the key processes behind the orientational ordering of azo-dye molecules are photochemically induced trans-cis isomerization and subsequent thermal and/or photochemical cis-trans back isomerization of azobenzene chromophores.

Owing to pronounced absorption dichroism of photoactive groups, the rate of the photoinduced isomerization strongly depends on orientation of the azo-dye molecules relative to the polarization vector of the actinic light, $\mathbf{E}_{\mathrm{UV}}$. So, the molecules with the optical transition dipole moment oriented perpendicular to $\mathbf{E}_{\mathrm{UV}}$ are almost inactive.

When the cis isomers are short living, the cis state becomes temporary populated during photoisomerization but reacts immediately back to the stable trans isomeric form. The trans-cis-trans isomerization cycles are accompanied by rotations of the azo-dye molecules that tend to minimize the absorption and become oriented along directions normal to the polarization vector of the exciting light $\mathbf{E}_{\mathrm{UV}}$. Nonphotoactive groups may then undergo reorientation due to cooperative motion $[17,19,33,35,36]$.

The above scenario, initially suggested in Ref. [13], is known as the regime of photo-orientation (angular redistribution) where the lifetime of cis isomers is short and POA is mainly due to the angular redistribution of the long axes of the trans molecules during the trans-cis-trans photoisomerization cycles. Note that, in the opposite case of long-living cis isomers, the regime of angular hole burning (photoselection) occurs so that the anisotropy is caused by angular selective burning of mesogenic trans isomers due to stimulated transitions to nonmesogenic cis form [22,35,37].

From the above it might be concluded that, whichever regime of the ordering takes place, the photoinduced orientational structure results from preferential alignment of azodye molecules along the directions perpendicular to the polarization vector of the actinic light, $\mathbf{E}_{\mathrm{UV}}$, determined by the dependence of the photoisomerization rate on the angle between $\mathbf{E}_{\mathrm{UV}}$ and the long molecular axis. So, it can be expected that the structure will be uniaxially anisotropic with the optical axis directed along the polarization vector.

Experimentally, this is, however, not the case. For example, constraints imposed by a medium may suppress outof-plane reorientation of the azobezene chromophores giving rise to the structures with strongly preferred in-plane alignment [23]. Another symmetry breaking effect induced by polymeric environment is that the photoinduced orientational structures can be biaxial $[21,23,35,38-40]$ (a recent review of medium effects on photochemical processes can be found in [41]).
It was recently found that, similar to the polymer systems, the long-term stable POA in the azo-dye SD1 films is characterized by the biaxial photoinduced structures with favored in-plane alignment [42]. Unlike azopolymers, photochromism in these films is extremely weak so that it is very difficult to unambiguously detect the presence of a noticeable fraction of cis isomers.

As compared to the polymer systems, modeling of photoinduced ordering in the azo-dye films has received little attention. In this paper we intend to fill the gap and describe the symmetry breaking and biaxiality effects using phenomenological models formulated on the basis of a unified approach to the kinetics of POA $[23,35]$. The layout of the paper is as follows.

In Sec. II A, we recapitulate the theory [35] by assuming that the azo-dye molecules can be represented by two-level molecular systems. This theoretical approach is based on the master equation combined with the kinetic equation for the additional (matrix) system, which phenomenologically accounts for the presence of long-living anisotropic (angular) correlations. Then, in Sec. II B, we discuss the relationship between the order parameter and the absorption tensors.

In Sec. II C, a phenomenological two-state model is introduced by specifying the angular redistribution probabilities and by expressing the order parameter correlation functions in terms of the order parameter tensor. In this model, the regime of photo-orientation with short-living excited state is characterized by weak photochromism and negligibly small fraction of excited molecules that rapidly decays after switching off irradiation.

According to Ref. [43], when the photochemical processes underlying photoisomerization are hindered, the process of photoinduced reorientation can be alternatively described as rotational diffusion of azo-dye molecules under the action of the polarized light.

In Sec. III A, we show that diffusion models of POA can be formulated as the free energy Fokker-Planck (FP) equation [44], describing light-induced reorientation of azo-dye molecules as rotational Brownian motion governed by the effective mean-field potential. Using this approach, the diffusion model suggested in [43] can be easily extended to the case of biaxial orientational structures. In Sec. III B, we introduce and study the simplified two-dimensional (2D) diffusion model that can be regarded as the first approximation representing the regime of purely in-plane reorientation.

The two-state and 2D diffusion models are employed to interpret the experimental data in Sec. IV. Finally, in Sec. V we present our results and make some concluding remarks. Technical details on solving the light transmission problem for a biaxially anisotropic absorbing layer and on using the analytical result to extract the extinction coefficients from the measured dependence of absorbance on the incidence angle are relegated to Appendix.

\section{MASTER EQUATION AND TWO-STATE MODELS}

In this section following the approach developed in Refs. $[23,35]$ we begin with the system of generalized kinetic rate equations that describe the azo-dye film in terms of one- 
particle distribution functions of an ensemble of two-level molecular systems. Mathematically, this system that will be referred to as the master equation can be regarded as a consequence of the Chapman-Kolmogorov equation [45]. The part of the system governed by the transitions between the ground and the excited states of azo-dye molecules determines two-state models characterized by the transition rates expressed in terms of the angular redistribution probabilities. We formulate our model by specifying the probabilities and then examine its predictions.

\section{A. Master equation}

We assume that azo-dye molecules are cylindrically symmetric and orientation of a molecule can be specified by the unit vector, $\hat{\mathbf{u}}=(\sin \theta \cos \phi, \sin \theta \sin \phi, \cos \theta)$, directed along the long molecular axis. So, for a spatially homogeneous system, angular distribution of the ground-state molecules at time $t$ is characterized by the number distribution function $N_{\mathrm{G}}(\hat{\mathbf{u}}, t)=V \rho_{\mathrm{G}}(\hat{\mathbf{u}}, t)$, where $V$ is the volume and $\rho_{\mathrm{G}}(\hat{\mathbf{u}}, t)$ is the one-particle distribution function describing the orientation-density profile of azo-dye molecules in the ground state. Similarly, for the azo-dye molecules in the excited state (excited molecules), the distribution function is $N_{\mathrm{E}}(\hat{\mathbf{u}}, t)=V \rho_{\mathrm{E}}(\hat{\mathbf{u}}, t)$.

Then the kinetic rate equations for $N_{\alpha}(\hat{\mathbf{u}}, t)$ can be written in the general form of master equation [45-47],

$$
\begin{aligned}
\frac{\partial N_{\alpha}}{\partial t}= & {\left[\frac{d N_{\alpha}}{d t}\right]_{\text {diff }}+\sum_{\beta \neq \alpha} \int\left[W\left(\alpha, \hat{\mathbf{u}} \mid \beta, \hat{\mathbf{u}}^{\prime}\right) N_{\beta}\left(\hat{\mathbf{u}}^{\prime}, t\right)\right.} \\
& \left.-W\left(\beta, \hat{\mathbf{u}}^{\prime} \mid \alpha, \hat{\mathbf{u}}\right) N_{\alpha}(\hat{\mathbf{u}}, t)\right] d \hat{\mathbf{u}}^{\prime} \\
& +\gamma_{\alpha}\left[N n_{\alpha}(t) f_{m}(\hat{\mathbf{u}}, t)-N_{\alpha}(\hat{\mathbf{u}}, t)\right], \quad \alpha, \beta \in\{\mathrm{G}, \mathrm{E}\},
\end{aligned}
$$

where $N$ is the total number of azo-dye molecules and $n_{\mathrm{G}}$ and $n_{\mathrm{E}}$ are the concentrations giving the number fractions of the ground-state and excited molecules, respectively.

The concentration $n_{\alpha}$ is defined through the relation linking the one-particle distribution function, $\rho_{\alpha}(\hat{\mathbf{n}}, t)$, and the corresponding normalized angular distribution functions, $f_{\alpha}(\hat{\mathbf{n}}, t)$,

$$
N_{\alpha}(\hat{\mathbf{u}}, t) \equiv V \rho_{\alpha}(\hat{\mathbf{u}}, t)=N n_{\alpha}(t) f_{\alpha}(\hat{\mathbf{u}}, t) .
$$

Note that the concentrations meet the relation

$$
n_{\mathrm{G}}(t)+n_{\mathrm{E}}(t)=1 \text {, }
$$

which is the conservation law for the total number of molecules.

The first term on the right-hand side of Eq. (1) is due to rotational diffusion of azo-dye molecules. It plays a leading part in the diffusion models that will be discussed later in Sec. III. In this section we concentrate on frictionless models representing the limiting case where the diffusion term is absent.

Now we specify the rate of the $\mathrm{G} \rightarrow \mathrm{E}$ transition stimulated by the incident UV light. Figure 1 shows that, in our frame of reference, the $y$ axis is directed along the polarization vector of the linearly polarized activating UV light, E

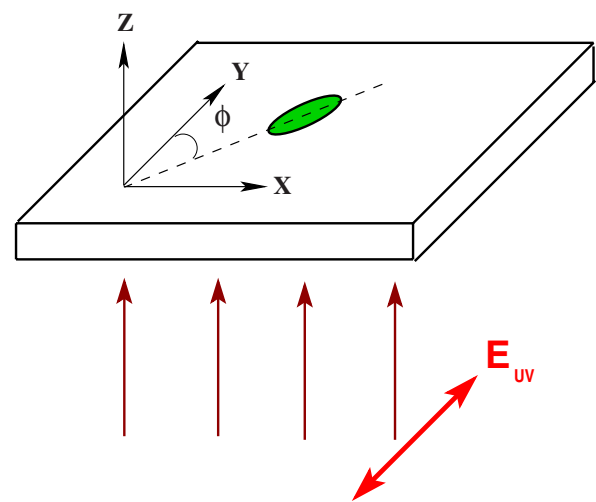

FIG. 1. (Color online) Frame of reference: the $z$ axis is normal to the substrate and the polarization vector of the activating light is directed along the $y$ axis.

$=E \hat{\mathbf{y}}$, the $z$ axis is normal to the substrates, and the unit vector $\hat{\mathbf{x}}=[\hat{\mathbf{y}} \times \hat{\mathbf{z}}]$ is parallel to the $x$ axis. So, the transition rate can be written as follows $[16,48]$ :

$$
\begin{aligned}
& W\left(\mathrm{E}, \hat{\mathbf{u}} \mid \mathrm{G}, \hat{\mathbf{u}}^{\prime}\right)=\Gamma_{e-g}\left(\hat{\mathbf{u}}, \hat{\mathbf{u}}^{\prime}\right) P_{g}\left(\hat{\mathbf{u}}^{\prime}\right), \\
P(\hat{\mathbf{u}})= & \left(\hbar \omega_{t}\right)^{-1} \Phi_{g \rightarrow e} \sum_{i, j} \sigma_{i j}(\hat{\mathbf{u}}) E_{i} E_{j}^{*} \\
= & q I_{\mathrm{UV}}\left(1+\sigma_{a} u_{y}^{2}\right) \\
= & \gamma_{p h}\left[3+\sigma_{a}+2 \sigma_{a} Q_{y y}(\hat{\mathbf{u}})\right] / 3, \quad \gamma_{p h}=q I_{\mathrm{UV}},
\end{aligned}
$$

where $\boldsymbol{\sigma}(\hat{\mathbf{u}})$ is the tensor of absorption cross section for the molecule in the ground state oriented along $\hat{\mathbf{u}}, \sigma_{i j}=\sigma_{\perp} \delta_{i j}$ $+\left(\sigma_{\|}-\sigma_{\perp}\right) u_{i} u_{j}, \sigma_{a} \equiv\left(\sigma_{\|}-\sigma_{\perp}\right) / \sigma_{\perp}$ is the absorption anisotropy parameter, $\hbar \omega_{t}$ is the photon energy, $\Phi_{g \rightarrow e}$ is the quantum yield of the process, $\Gamma_{g-e}\left(\hat{\mathbf{u}}, \hat{\mathbf{u}}^{\prime}\right)$ describes the angular redistribution of the molecules in the excited state, $I_{\mathrm{UV}}$ is the pumping intensity, and $q \equiv\left(\hbar \omega_{t}\right)^{-1} \Phi_{g \rightarrow e} \sigma_{\perp}^{(g)}$. Similarly, the rate of the spontaneous $\mathrm{E} \rightarrow \mathrm{G}$ transition is as follows:

$$
W\left(\mathrm{G}, \hat{\mathbf{u}} \mid \mathrm{E}, \hat{\mathbf{u}}^{\prime}\right)=\gamma_{e} \Gamma_{g-e}\left(\hat{\mathbf{u}}, \hat{\mathbf{u}}^{\prime}\right),
$$

where $\gamma_{e} \equiv 1 / \tau_{e}$ and $\tau_{e}$ is the lifetime of the excited state, which is expressed in terms of the angular redistribution probability $\Gamma_{g-e}\left(\hat{\mathbf{u}}, \hat{\mathbf{u}}^{\prime}\right)$. All the angular redistribution probabilities are normalized so as to meet the standard normalization condition for probability densities,

$$
\int \Gamma_{\beta-\alpha}\left(\hat{\mathbf{u}}, \hat{\mathbf{u}}^{\prime}\right) d \hat{\mathbf{u}}=1,
$$

where $d \hat{\mathbf{u}} \equiv \sin \theta d \theta d \phi$.

In Eq. (1), the presence of long-living angular correlations coming from anisotropic interactions between azo-dye molecules and collective modes of confining environment is taken into account by using the phenomenological approach suggested in Refs. [35,40]. In this approach, the effective anisotropic field, which results in the long-term stability effect and determines angular distribution of the molecules in the stationary regime, is introduced through the additional angular distribution function, $f_{m}(\hat{\mathbf{u}}, t)$, characterizing the additional subsystem that, for brevity, will be referred to as the matrix system. 
It bears close resemblance to the equilibrium distribution of the mean-field theories of photoinduced optical anisotropy $[31,32,34]$. In these theories, this distribution has been assumed to be proportional to $\exp \left[-V(\hat{\mathbf{u}}) / k_{B} T\right]$, where $V(\hat{\mathbf{u}})$ is the mean-field potential that depends on the order parameter tensor.

The last square bracketed term on the right-hand side of Eq. (1) describes the process that equilibrates the absorbing molecules and the matrix system in the absence of irradiation. The equilibrium angular distributions $f_{\mathrm{G}}^{(e q)}$ and $f_{\mathrm{E}}^{(e q)}$ are both equal to $f_{m}$.

The latter is the case for the mean-field models considered in $[31,32,34]$. In these models the excited molecules (cis fragments) are assumed to be long living with $\gamma_{e}=0$ and $\gamma_{\mathrm{G}}=\gamma_{\mathrm{E}}$. We can now recover the models by setting the angular redistribution probabilities $\Gamma_{g-e}\left(\hat{\mathbf{u}}, \hat{\mathbf{u}}^{\prime}\right)$ and $\Gamma_{e-g}\left(\hat{\mathbf{u}}, \hat{\mathbf{u}}^{\prime}\right)$ equal to the equilibrium distribution, $f_{m}=p(\hat{\mathbf{u}})$, determined by the mean-field potential $V(\hat{\mathbf{u}}): p(\hat{\mathbf{u}}) \propto \exp \left(-V / k_{B} T\right)$. So, the mean-field approach introduces the angular redistribution operators acting as projectors onto the angular distribution of the matrix system. This is the order parameter dependent distribution that characterizes orientation of the azomolecules after excitation.

An alternative and more general approach is to determine the distribution function $f_{m}(\hat{\mathbf{u}}, t)$ from the kinetic equation that can be written in the following form [35]:

$$
\frac{\partial f_{m}(\hat{\mathbf{u}}, t)}{\partial t}=-\sum_{\alpha=\{\mathrm{G}, \mathrm{E}\}} \gamma_{m}^{(\alpha)} n_{\alpha}(t)\left[f_{m}(\hat{\mathbf{u}}, t)-f_{\alpha}(\hat{\mathbf{u}}, t)\right] .
$$

Using system (1) and relations (4)-(6) it is not difficult to deduce the equation for $n_{\mathrm{G}}(t)$,

$$
\frac{\partial n_{\mathrm{G}}}{\partial t}=\gamma_{e}\left(1-n_{\mathrm{G}}\right)-\langle P\rangle_{\mathrm{G}} n_{\mathrm{G}},
$$

where the angular brackets $\langle\cdots\rangle_{\alpha}$ stand for averaging over the angles with the distribution function $f_{\alpha}$. Owing to condition (7), this equation does not depend on the form of the angular redistribution probabilities.

Equations for the angular distribution functions $f_{\mathrm{G}}(\hat{\mathbf{u}}, t)$ and $f_{\mathrm{E}}(\hat{\mathbf{u}}, t)$ can be derived from Eq. (1) by using relations (4)-(9). The result is as follows:

$$
\begin{aligned}
n_{\mathrm{E}} \frac{\partial f_{\mathrm{E}}}{\partial t}= & -n_{\mathrm{G}}\left[\langle P\rangle_{\mathrm{G}} f_{\mathrm{E}}-\int \Gamma_{e-g}\left(\hat{\mathbf{u}}, \hat{\mathbf{u}}^{\prime}\right) P\left(\hat{\mathbf{u}}^{\prime}\right) f_{\mathrm{G}}\left(\hat{\mathbf{u}}^{\prime}, t\right) d \hat{\mathbf{u}}^{\prime}\right] \\
& -\gamma_{\mathrm{E}} n_{\mathrm{E}}\left[f_{\mathrm{E}}-f_{m}\right] \\
n_{\mathrm{G}} \frac{\partial f_{\mathrm{G}}}{\partial t}= & -n_{\mathrm{G}}\left[P(\hat{\mathbf{u}})-\langle P\rangle_{\mathrm{G}}\right] f_{\mathrm{G}} \\
& +\gamma_{e} n_{\mathrm{E}} \int \Gamma_{g-e}\left(\hat{\mathbf{u}}, \hat{\mathbf{u}}^{\prime}\right) f_{\mathrm{E}}\left(\hat{\mathbf{u}}^{\prime}, t\right) d \hat{\mathbf{u}}^{\prime}-\gamma_{e} n_{\mathrm{E}} f_{\mathrm{G}} \\
& -\gamma_{\mathrm{G}} n_{\mathrm{G}}\left[f_{\mathrm{G}}-f_{m}\right] .
\end{aligned}
$$

In Sec. II C, the system of Eqs. (9)-(11) will be used as the starting point to introduce our two-state phenomenological model of POA. This model is formulated as the kinetic equations for the orientational order parameters that will be dis- cussed in Sec. II B where the fraction of the excited molecules is tacitly assumed to be negligibly small.

\section{B. Order parameters and absorption tensor}

Quadrupolar orientational ordering of rodlike molecules is characterized using the traceless symmetric second-rank tensor [49],

$$
\mathbf{Q}(\hat{\mathbf{u}})=(3 \hat{\mathbf{u}} \otimes \hat{\mathbf{u}}-\mathbf{I}) / 2,
$$

where I is the identity matrix. Dyadic (12) can be averaged over orientation of molecules with the normalized angular distribution function $f(\hat{\mathbf{u}})$ to yield the order parameter tensor $\mathbf{S}$,

$$
\langle\mathbf{Q}\rangle=\int \mathbf{Q}(\hat{\mathbf{u}}) f(\hat{\mathbf{u}}) d \hat{\mathbf{u}}=\mathbf{S},
$$

where $d \hat{\mathbf{u}} \equiv \sin \theta d \theta d \phi$. In our case, on symmetry grounds, it can be expected that the basis vectors $\{\hat{\mathbf{x}}, \hat{\mathbf{y}}, \hat{\mathbf{z}}\}$ shown in Fig. 1 define the principal axes of the order parameter tensor. So, tensor (13) is given by

$$
\mathbf{S}=\operatorname{diag}\left(S_{x}, S_{y}, S_{z}\right) .
$$

The dielectric tensor, $\boldsymbol{\varepsilon}$, can also be written in the diagonal form,

$$
\varepsilon=\operatorname{diag}\left(\epsilon_{x}, \epsilon_{y}, \epsilon_{z}\right), \quad \varepsilon_{\alpha \beta}=\epsilon_{\alpha} \delta_{\alpha \beta} .
$$

In the presence of absorption, tensor (15) is complex valued and its principal values, $\left\{\epsilon_{x}, \epsilon_{y}, \epsilon_{z}\right\}$, are expressed in terms of the refractive indices, $\left\{n_{x}^{(r)}, n_{y}^{(r)}, n_{z}^{(r)}\right\}$, and the extinction coefficients, $\left\{\kappa_{x}, \kappa_{y}, \kappa_{z}\right\}$, as follow [50]:

$$
\epsilon_{\alpha}=\epsilon_{\alpha}^{\prime}+i \epsilon_{\alpha}^{\prime \prime}, \quad \mu \epsilon_{\alpha}=n_{\alpha}^{2}=\left(n_{\alpha}^{(r)}+i \kappa_{\alpha}\right)^{2} .
$$

We can now define the absorption order parameters through the relation

$$
S_{i}^{(a)}=\frac{2 \kappa_{i}-\kappa_{j}-\kappa_{k}}{2\left(\kappa_{i}+\kappa_{j}+\kappa_{k}\right)}=\frac{2 D_{i}^{(a)}-D_{j}^{(a)}-D_{k}^{(a)}}{2\left(D_{i}^{(a)}+D_{j}^{(a)}+D_{k}^{(a)}\right)}, \quad i \neq j \neq k,
$$

where the optical densities $\left\{D_{x}^{(a)}, D_{y}^{(a)}, D_{z}^{(a)}\right\}$ are proportional to the extinction coefficients: $D_{i}^{(a)} \propto \kappa_{i}$. Note that the optical density $D_{\|}^{(a)} \equiv D_{y}^{(a)}\left[D_{\perp}^{(a)} \equiv D_{x}^{(a)}\right]$ can be determined experimentally by measuring the absorption coefficient for a testing beam which is propagating along the normal to the film substrate (the $z$ axis) and is linearly polarized parallel [perpendicular] to the polarization vector of the activating UV light (the $y$ axis).

Now, following Refs. [30,40], we touch briefly on the relation between the orientational and the absorption order parameters defined in Eqs. (14) and (17), respectively. To this end, we begin with the absorption tensor of an azo-dye molecule,

$$
\sigma_{i j}(\hat{\mathbf{u}})=\sigma_{\perp} \delta_{i j}+\left(\sigma_{\|}-\sigma_{\perp}\right) u_{i} u_{j},
$$

which is assumed to be uniaxially anisotropic. Its orientational average takes the following matrix form:

$$
\langle\boldsymbol{\sigma}\rangle=\left(\sigma_{\mathrm{av}} \mathbf{I}+2 \Delta \sigma \mathbf{S}\right) / 3,
$$




$$
\sigma_{\mathrm{av}}=\sigma_{\|}+2 \sigma_{\perp}, \quad \Delta \sigma=\sigma_{\|}-\sigma_{\perp},
$$

where the angular brackets $\langle\cdots\rangle$ denote orientational averaging [see Eq. (13)].

In the low concentration approximation, the optical densities are proportional to the corresponding components of tensor (19),

$$
\begin{gathered}
D_{\|}^{(a)}=D_{x}^{(a)} \propto \rho\left(\sigma_{\mathrm{av}}+2 \Delta \sigma S_{x}\right) / 3, \\
D_{\perp}^{(a)}=D_{y}^{(a)} \propto \rho\left(\sigma_{\mathrm{av}}+2 \Delta \sigma S_{y}\right) / 3, \\
D_{z}^{(a)} \propto \rho\left(\sigma_{\mathrm{av}}+2 \Delta \sigma S_{z}\right) / 3,
\end{gathered}
$$

and on substituting the expressions for the optical densities [Eq. (21)] into Eq. (17) we obtain

$$
S_{i}^{(a)}=r_{a} S_{i}, \quad r_{a}=\sigma_{a} /\left(3+\sigma_{a}\right),
$$

where $\sigma_{a}=\Delta \sigma / \sigma_{\perp}$. So, the absorption order parameters [Eq. (17)] are equal to the corresponding elements of order parameter tensor (14) only in the limiting case where absorption of waves propagating along the long molecular axis is negligibly small: $\sigma_{\perp} \rightarrow 0$ and $\sigma_{\mathrm{av}}=3 \sigma_{\perp}+\Delta \sigma \rightarrow \Delta \sigma$. Note that the average optical density $D_{x}^{(a)}+D_{y}^{(a)}+D_{z}^{(a)}$ is proportional to $\rho \sigma_{\mathrm{av}}$ and thus typically does not depend on the irradiation dose.

\section{Two-state model}

We can now present our two-state model. Following the line of reasoning from Refs. [35,40], we assume that the angular redistribution probabilities, $\Gamma_{e-g}$ and $\Gamma_{g-e}$, are both isotropic,

$$
\Gamma_{e-g}\left(\hat{\mathbf{u}}, \hat{\mathbf{u}}^{\prime}\right)=\Gamma_{g-e}\left(\hat{\mathbf{u}}, \hat{\mathbf{u}}^{\prime}\right)=\frac{1}{4 \pi} \equiv f_{\text {iso }}
$$

and

$$
\gamma_{\mathrm{E}}=\gamma_{m}^{(\mathrm{E})}=0,
$$

so that the anisotropic field represented by $f_{m}$ does not influence the angular distribution of excited molecules. Equilibrium properties of excited and ground-state molecules are thus characterized by two different equilibrium angular distributions: $f_{\text {iso }}$ and $f_{m}$, respectively.

On substituting relations (23) and (24) into Eqs. (8) and (11) we deduce the following system of kinetic equations for the order parameters:

$$
\begin{aligned}
& n_{\mathrm{G}} \frac{\partial S_{i j}^{(\mathrm{G})}}{\partial t}=-\frac{2 \sigma_{a} \gamma_{p h}}{3} n_{\mathrm{G}} G_{i j ; y y}^{(\mathrm{G})}-\gamma_{e}\left(1-n_{\mathrm{G}}\right) S_{i j}^{(\mathrm{G})} \\
&+\gamma_{\mathrm{G}} n_{\mathrm{G}}\left(S_{i j}^{(m)}-S_{i j}^{(\mathrm{G})}\right), \\
& \frac{\partial S_{i j}^{(m)}}{\partial t}=-\gamma_{m} n_{\mathrm{G}}\left(S_{i j}^{(m)}-S_{i j}^{(\mathrm{G})}\right),
\end{aligned}
$$

where $G_{i j ; m n}^{(\mathrm{G})}$ is the order parameter correlation function given by

$$
G_{i j ; m n}^{(\mathrm{G})}=\left\langle Q_{i j}(\hat{\mathbf{u}}) Q_{m n}(\hat{\mathbf{u}})\right\rangle_{\mathrm{G}}-S_{i j}^{(\mathrm{G})} S_{m n}^{(\mathrm{G})}
$$

The key point of the approach suggested in Ref. [35] is the assumption that the correlators [Eq. (26)], which characterize response of azo-dye molecules to the pumping light and enter the kinetic equations for the order parameter components [Eq. (25a)], can be expressed in terms of the averaged order parameters $S_{i j}^{(\mathrm{G})}$. It was also shown that the parabolic approximation used in Ref. [23] can be improved by rescaling the order parameter components $S_{i i}^{(\mathrm{G})} \rightarrow \lambda S_{i i}^{(\mathrm{G})}$ with $\lambda=(1+0.6 \sqrt{30}) / 7$, computed from the condition that there are no fluctuations provided the molecules are perfectly aligned along the coordinate unit vector $\hat{\mathbf{e}}_{i}: G_{i i ; i i}^{(\mathrm{G})}=0$ at $S_{i i}^{(\mathrm{G})}$ $=1$. In Ref. [35] this heuristic procedure has also been found to provide a reasonably accurate approximation for the correlators calculated by assuming that the angular distribution of molecules can be taken in the form of distribution functions used in the variational mean-field theories of liquid crystals $[49,51]$.

So, the resulting system of equations that govern temporal evolution of the order parameters reads

$$
\begin{aligned}
& n_{\mathrm{G}} \frac{\partial S}{\partial t}= \frac{2 \sigma_{a} \gamma_{p h}}{3} n_{\mathrm{G}}\left(5 / 7+2 \lambda / 7 S-\lambda^{2} S^{2}\right) \\
&-\gamma_{e}\left(1-n_{\mathrm{G}}\right) S+\gamma_{\mathrm{G}} n_{\mathrm{G}}\left(S_{m}-S\right), \\
& n_{\mathrm{G}} \frac{\partial \Delta S}{\partial t}=-\frac{2 \sigma_{a} \gamma_{p h}}{3} n_{\mathrm{G}} \lambda(2 / 7+\lambda S) \Delta S \\
&-\gamma_{e}\left(1-n_{\mathrm{G}}\right) \Delta S+\gamma_{\mathrm{G}} n_{\mathrm{G}}\left(\Delta S_{m}-\Delta S\right), \\
& \frac{\partial S_{m}}{\partial t}=-\gamma_{m} n_{\mathrm{G}}\left(S_{m}-S\right), \\
& \frac{\partial \Delta S_{m}}{\partial t}=-\gamma_{m} n_{\mathrm{G}}\left(\Delta S_{m}-\Delta S\right),
\end{aligned}
$$

where $\gamma_{m} \equiv \gamma_{m}^{(\mathrm{G})}, \quad S \equiv S_{x x}^{(\mathrm{G})}, \Delta S \equiv S_{y y}^{(\mathrm{G})}-S_{z z}^{(\mathrm{G})}, \quad S_{m} \equiv S_{x x}^{(m)}$, and $\Delta S_{m} \equiv S_{y y}^{(m)}-S_{z z}^{(m)}$.

\section{Long term stability and photosteady state}

Mathematically, the two-state model is defined by equations for the order parameters and the concentration given in Eqs. (27) and (9), respectively. We may now pass on to discussing some of its general properties.

Our first remark concerns the effect of the long-term stability of POA. It means that there is the amount of the photoinduced anisotropy preserved intact for long time after switching off the light. Clearly, this is a memory effect and the system does not relax back to the off state characterized by irradiation independent equilibrium values of the order parameters.

In order to see how this effect is described in our model, we assume that the activating light is switched off at time $t$ $=t_{\text {off }}$ and consider subsequent evolution of the order param-

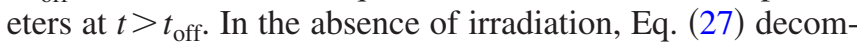
poses into two decoupled identical systems of equations for two pairs of the order parameters: $\left\{S, S_{m}\right\}$ and $\left\{\Delta S, \Delta S_{m}\right\}$. So, without the loss of generality we may restrict ourselves to the evolution of the $x$ components of order parameters, 
$\left\{S, S_{m}\right\} \equiv\left\{S_{x x}^{(\mathrm{G})}, S_{x x}^{(m)}\right\}$, governed by the equations

$$
\begin{gathered}
\frac{\partial S}{\partial t}=-\gamma_{e}\left(1 / n_{\mathrm{G}}-1\right) S+\gamma_{\mathrm{G}}\left(S_{m}-S\right), \\
\frac{\partial S_{m}}{\partial t}=-\gamma_{m}\left(S_{m}-S\right)
\end{gathered}
$$

supplemented with the initial conditions

$$
S\left(t_{\text {off }}\right)=S_{\text {off }}, \quad S_{m}\left(t_{\text {off }}\right)=S_{\text {off }}^{(m)} .
$$

At $I_{\mathrm{UV}}=0$, equation for concentration (9) is easy to solve. So, for the initial value problem with $n_{\mathrm{G}}\left(t_{\text {off }}\right)=n_{\text {off }}$, we have

$$
1-n_{\mathrm{G}}=\exp \left(-\gamma_{e} \Delta t\right)\left(1-n_{\text {off }}\right), \quad \Delta t=t-t_{\text {off }} .
$$

From Eq. (31) it is clear that, in the limiting case of shortliving excited state with $\Delta t \gg 1 / \gamma_{e}$, the concentration of excited molecules, $n_{\mathrm{E}}=1-n_{\mathrm{G}}$, rapidly decays to zero. In this regime, the first term on the right-hand side of Eq. (28) is negligibly small and can be disregarded. Equations (32) and (33) can now be easily solved to yield the formulas

$$
\begin{gathered}
S(t)=S_{\text {off }}^{(\text {st })}+\gamma_{\mathrm{G}}\left[S_{\text {off }}-S_{\text {off }}^{(m)}\right] \exp (-\gamma \Delta t) / \gamma, \\
S_{m}(t)=S_{\text {off }}^{(\text {st) }}-\gamma_{m}\left[S_{\text {off }}-S_{\text {off }}^{(m)}\right] \exp (-\gamma \Delta t) / \gamma,
\end{gathered}
$$

where $\gamma=\gamma_{m}+\gamma_{\mathrm{G}}$ and

$$
S_{\text {off }}^{(\text {st) }}=\left(\gamma_{m} S_{\text {off }}+\gamma_{\mathrm{G}} S_{\text {off }}^{(m)}\right) / \gamma .
$$

Evidently, the order parameters defined in Eqs. (32) and (33) evolve in time approaching stationary value (34). The memory effect manifests itself in the dependence of the stationary order parameter, $S_{\mathrm{off}}^{(\mathrm{st})}$, on the (initial) conditions [Eq. (30)] at the instant the activating light is switched off.

The photosteady states reached in the long irradiation time limit are represented by stationary solutions of the system [Eq. (25)] and the concentration [Eq. (9)].

The steady state concentration of excited molecules can be expressed in terms of the steady state order parameter, $S_{y y}^{(\text {st) }}$, through the relation

$$
1-n_{\mathrm{G}}^{(\mathrm{st})}=\frac{3+\sigma_{a}\left(1+2 S_{y y}^{(\mathrm{st})}\right)}{3(r+1)+\sigma_{a}\left(1+2 S_{y y}^{(\mathrm{st})}\right)},
$$

where $r \equiv \gamma_{e} / \gamma_{p h}$. From Eq. (35) it can be seen that, in the case where the lifetime of the excited state is short and the ratio $r$ is large, $r \gg 1$, the fraction of the excited molecules is negligible, so that $n_{\mathrm{G}}^{\text {(st) }} \approx 1$.

On substituting Eq. (5) into the steady state relation

$$
\left\langle Q_{i j}(\hat{\mathbf{u}}) P(\hat{\mathbf{u}})\right\rangle_{\mathrm{G}}=0
$$

derived from Eqs. (25a) and (9) we obtain equation for the steady state order parameters,

$$
S_{i i}^{(\mathrm{st})}=-\frac{2 \sigma_{a}}{3+\sigma_{a}}\left\langle Q_{i i}(\hat{\mathbf{u}}) Q_{y y}(\hat{\mathbf{u}})\right\rangle_{\mathrm{G}} .
$$

From Eq. (25b) the difference between the order parameters of the matrix system and the ground-state azo-dye molecules dies out as the photosteady state is approached, $S_{i j}^{(\mathrm{G})}-S_{i j}^{(m)}$ $\rightarrow 0$ at $t \rightarrow \infty$. Interestingly, Eq. (37) shows that the order parameters, $S_{i i}^{(\mathrm{st})}$, characterizing the regime of photosaturation, are independent of the light intensity, $I_{\mathrm{UV}}$.

For the specific form of the correlators used to obtain system (27), the photosteady state is uniaxial with $S_{y y}^{(\mathrm{st})}$ $=S_{z z}^{(\mathrm{st})}$ and the $x$ component of the order parameter tensor, $S_{\mathrm{st}}=S_{x x}^{(\mathrm{st})}$, can be found by solving the equation

$$
2 \sigma_{a}\left(1 / 5+2 \lambda / 7 S_{\mathrm{st}}-\lambda^{2} S_{\mathrm{st}}^{2}\right)=S_{\mathrm{st}}\left[3+\sigma_{a}\left(1+2 S_{\mathrm{st}}\right)\right] .
$$

\section{NONLINEAR FOKKER-PLANCK EQUATIONS AND DIFFUSION MODEL}

The two-state model introduced in Sec. II Ccan be regarded as a frictionless model driven by the transitions between the ground and excited states. A peculiarity of this model is that the excited molecules have no direct effect on ordering dynamics of the ground-state molecules and, in the regime of short-living excited state with $\gamma_{p h} / \gamma_{e} \ll 1$, the fraction of the excited molecules can be too small to reveal itself in experiments.

Since, in the azo-dye films, the nature of the excited state remains unknown, it is reasonable to consider diffusion models that do not involve excited molecules. Such a model was previously suggested in Ref. [43].

In this section we extend the diffusion model [43] by using the approach based on FP equations [52]. For our purposes, of particular interest are the so-called nonlinear meanfield FP equations determined by the effective free energy functional, $F[P]$, as follows

$$
\partial_{t} P=\partial_{i}\left\{P \partial_{i} \frac{\delta F[P]}{\delta P}\right\} \equiv \nabla \cdot\left\{P \nabla \frac{\delta F[P]}{\delta P}\right\},
$$

where $\partial_{i} \equiv \partial / \partial x_{i}$ and $P \equiv P(\mathbf{x}, t)$ is the probability density (distribution function). The theory and applications of such equations representing the special case of nonlinear FP equations were recently reviewed in the monograph [44].

In Sec. III A, we show that the rotational diffusion model governed by the mean-field potential can be derived from the mean-field FP equation extended to the case of the rotational diffusion.

\section{A. Rotational mean-field Fokker-Planck equations}

In diffusion models, reorientation of molecules is described as the rotational Brownian motion. So, our first step is to recast the mean-field FP equation [Eq. (39)] into the form of the rotational mean-field FP equation,

$$
\partial_{t} f=-\mathcal{J}_{i} D_{i j}^{(\mathrm{rot})}\left\{f \mathcal{J}_{j} \frac{\delta F[f]}{\delta f}\right\} \equiv-\mathcal{J} \cdot \mathbf{D}_{\text {rot }}\left\{f \cdot \mathcal{J} \frac{\delta F[f]}{\delta f}\right\},
$$

where $D_{i j}^{(\text {rot })}$ is the rotational diffusion tensor and the components of the angular momentum operator, $\mathcal{J}$, expressed in terms of the Euler angles, $\boldsymbol{\omega} \equiv(\alpha, \beta, \gamma)$, are [53] $\mathcal{J}_{x}=-i\left\{-\cos \alpha \cot \beta \partial_{\alpha}-\sin \alpha \partial_{\beta}+\frac{\cos \alpha}{\sin \gamma} \partial_{\gamma}\right\}, \quad \mathcal{J}_{y}$ $=-i\left\{-\sin \alpha \cot \beta \partial_{\alpha}+\cos \alpha \partial_{\beta}+\frac{\sin \alpha}{\sin \gamma} \partial_{\gamma}\right\}$, and $\mathcal{J}_{z}=-i \partial_{\alpha}$. Note that, algebraically, Eq. (40) can be regarded as a modified 
version of the FP equation [Eq. (39)], where the nabla operator $-i \boldsymbol{\nabla}$ is replaced by the angular momentum operator $\mathcal{J}$, thus changing the generators of spatial translations to the ones of rotations $[54,55]$.

When the effective free energy functional is a sum of two terms that represent the contributions coming from the effective internal energy, $U[f]$, and the Boltzmann entropy

$$
F[f]=U[f]+\langle\ln f\rangle,
$$

the variational derivative of the free energy takes the form

$$
\frac{\delta F}{\delta f}=V+\ln f+1, \quad V=\frac{\delta U}{\delta f},
$$

where $V$ is the mean-field potential. On substituting relation (42) into Eq. (40) we obtain the mean-field FP equation,

$$
\partial_{t} f=-\mathcal{J} \cdot \mathbf{D}_{\text {rot }}\{\mathcal{J} f+f \cdot \mathcal{J} V\},
$$

describing the rotational diffusion governed by mean-field potential (42).

The rotational mean-field FP equation [Eq. (43)] is linear provided that the potential, $V$, is independent of the angular distribution function, $f$. This is the special case corresponding to the well-known rotational diffusion equation that has been widely used to study a large variety of problems based on the rotational diffusion model for the rotational motion of molecules in the presence of external fields. These include dielectric and Kerr effect relaxation of polar liquids [56-63], rotational diffusion of a probe molecule dissolved in a liquid crystal phase [64-69], and molecular reorientation in liquid crystal phases [70-73].

When molecules and the orientational distribution function are cylindrically symmetric, the model can be described in terms the angle between the electric field and the molecular axis [56-59], whereas angular distributions of a more general form require using both azimuthal and polar angles that characterize orientation of the molecules $[60,61]$. In this case, for uniaxial (rodlike) molecules, the distribution function $f(\alpha, \beta, \gamma) \equiv f(\boldsymbol{\omega})$ is independent of the Euler angle $\gamma$, $f(\boldsymbol{\omega})=f(\phi, \theta) \equiv f(\hat{\mathbf{u}})$, and the angular momentum operator can be expressed in terms of the azimuthal and zenithal (polar) angles, $\phi$ and $\theta$, as follows:

$$
i \mathcal{J} \stackrel{\partial_{\gamma} \rightarrow 0}{\rightarrow} i \mathbf{L}=[\mathbf{r} \times \boldsymbol{\nabla}]=\hat{\mathbf{e}}_{\phi} \partial_{\theta}-[\sin \theta]^{-1} \hat{\mathbf{e}}_{\theta} \partial_{\phi},
$$

where $\quad \hat{\mathbf{e}}_{\theta}=(\cos \theta \cos \phi, \cos \theta \sin \phi,-\sin \theta) \quad$ and $\quad \hat{\mathbf{e}}_{\phi}$ $=(-\sin \phi, \cos \phi, 0)$.

A more complicated biaxial case occurs for asymmetric top molecules $[62,72]$, macromolecules in liquid solutions [63], and probes in the biaxial liquid crystal phase [67]. For such low symmetry, analytical treatment cannot be simplified and involves the three Euler angles, $\boldsymbol{\omega} \equiv(\alpha, \beta, \gamma)$.

In the lowest order approximation, nonlinearity can be introduced through the truncated expansion for the internal energy functional $U[f]$ retaining one-particle (linear) and two-particle (quadratic) terms,

$$
\begin{aligned}
U[f]= & \int U_{1}(\boldsymbol{\omega}) f(\boldsymbol{\omega}) d \boldsymbol{\omega} \\
& +\frac{1}{2} \int f\left(\boldsymbol{\omega}_{1}\right) U_{2}\left(\boldsymbol{\omega}_{1}, \boldsymbol{\omega}_{2}\right) f\left(\boldsymbol{\omega}_{2}\right) d \boldsymbol{\omega}_{1} d \boldsymbol{\omega}_{2},
\end{aligned}
$$

where $\int d \boldsymbol{\omega} \cdots=\int_{0}^{2 \pi} d \alpha \int_{0}^{\pi} \sin \beta d \beta \int_{0}^{2 \pi} d \gamma \cdots$ and $U_{2}\left(\boldsymbol{\omega}_{1}, \boldsymbol{\omega}_{2}\right)$ $=U_{2}\left(\boldsymbol{\omega}_{2}, \boldsymbol{\omega}_{1}\right)$ is the symmetrized two-particle kernel. The effective potential

$$
V(\boldsymbol{\omega})=\frac{\delta U}{\delta f(\boldsymbol{\omega})}=U_{1}(\boldsymbol{\omega})+\int U_{2}\left(\boldsymbol{\omega}, \boldsymbol{\omega}^{\prime}\right) f\left(\boldsymbol{\omega}^{\prime}\right) d \boldsymbol{\omega}^{\prime}
$$

is the sum of the external field potential, $U_{1}(\boldsymbol{\omega})$, and the contribution coming from the two-particle intermolecular interactions.

For rodlike azo-dye molecules, the one-particle part of effective potential (46) can be written as a sum of the two terms,

$$
U_{1}(\hat{\mathbf{u}})=U_{I}(\hat{\mathbf{u}})+U_{s}(\hat{\mathbf{u}}),
$$

where the light-induced contribution

$$
U_{I}(\hat{\mathbf{u}})=u_{I} \mathbf{E}_{\mathrm{UV}}^{*} \cdot \mathbf{Q}(\hat{\mathbf{u}}) \cdot \mathbf{E}_{\mathrm{UV}}=u_{I} I_{\mathrm{UV}} Q_{y y}(\hat{\mathbf{u}})
$$

comes from the interaction of azo-molecules with the activating UV light and the surface-induced potential

$$
U_{s}(\hat{\mathbf{u}})=u_{s} \hat{\mathbf{z}} \cdot \mathbf{Q} \cdot \hat{\mathbf{z}}=u_{s} Q_{z z}(\hat{\mathbf{u}})
$$

takes into account conditions at the bounding surfaces of the azo-dye layer.

Assuming that the two-particle interaction is of the MaierSaupe form,

$$
U_{2}\left(\hat{\mathbf{u}}_{1}, \hat{\mathbf{u}}_{2}\right)=u_{2} \operatorname{Tr}\left[\mathbf{Q}\left(\hat{\mathbf{u}}_{1}\right) \cdot \mathbf{Q}\left(\hat{\mathbf{u}}_{2}\right)\right]=u_{2} Q_{i j}\left(\hat{\mathbf{u}}_{1}\right) Q_{i j}\left(\hat{\mathbf{u}}_{2}\right),
$$

we derive the expression for the effective potential of azodye molecules,

$$
V(\hat{\mathbf{u}})=u_{I} I_{\mathrm{UV}} Q_{y y}(\hat{\mathbf{u}})+u_{s} Q_{z z}(\hat{\mathbf{u}})+u_{2} S_{i j} Q_{i j}(\hat{\mathbf{u}}) .
$$

Generally, the equilibrium angular distribution can be obtained as a stationary solution to the FP equation [Eq. (43)]. It is not difficult to see that the stationary solution given by

$$
f_{\mathrm{st}}(\boldsymbol{\omega})=Z_{\mathrm{st}}^{1} \exp [-V(\boldsymbol{\omega})], \quad Z_{\mathrm{st}}=\int \exp [-V(\boldsymbol{\omega})] d \boldsymbol{\omega}
$$

is the Boltzmann distribution determined by the effective potential. Note that the internal energy and the potential defined in Eqs. (41) and (42) are dimensionless. The energy scale is determined by the temperature factor $k_{B} T$ incorporated into the rotation diffusion tensor.

When the FP equation is linear, stationary distribution (52) describing the equilibrium state is unique. In contrast to the linear case, effective potential (51) depends on the elements of the averaged orientational order parameter tensor (13): $V(\boldsymbol{\omega})=V(\hat{\mathbf{u}} \mid \mathbf{S})$. So, the components of the order parameter tensor in the stationary state, $S_{i j}=S_{i j}^{(\mathrm{st})}$, can be found from the self-consistency condition 


$$
S_{i j}=\int Q_{i j}(\hat{\mathbf{u}}) f_{\mathrm{st}}(\hat{\mathbf{u}} \mid \mathbf{S}) d \hat{\mathbf{u}} .
$$

In general, there are several solutions of Eq. (53) representing multiple local extrema (stationary points) of the free energy

$$
F\left[f_{\mathrm{st}}\right] \equiv F_{\mathrm{st}}(\mathbf{S})=-\frac{u_{2}}{2} S_{i j} S_{i j}-\ln Z_{\mathrm{st}}(\mathbf{S}) .
$$

Following the line of reasoning presented in Ref. [44] and using effective free energy (41) as the Lyapunov functional, it is not difficult to prove the $H$ theorem for nonlinear FP equations of form (40). It follows that all transient solutions converge to stationary ones in the long time limit. So, each stable stationary distribution is characterized by the basin of attraction giving orientational states (angular distributions) that evolve in time approaching the stationary distribution.

Free energy FP equations (both linear and nonlinear) are generally not exactly solvable. So, we conclude this section with remarks on numerical methods applicable to nonlinear FP equations.

The method based on distributed approximating functionals (DAFs), which couples the path-integral concept to the DAF idea, is proposed for numerically solving a general class of nonlinear time-dependent Fokker-Planck (FP) equations in [74]. The approach is applied to solve a nonlinear self-consistent dynamic mean-field problem for which both the cumulant expansion and the scaling theory have been found by Drozdov and Morillo [75] to be inadequate to describe a long-lived transient bimodality.

In Ref. [75], a finite-difference method for solving a general class of linear and nonlinear FP equations based on a $K$-point Stirling interpolation formula is suggested. A procedure to systematically evaluate all the moments of the FP equation by expanding them in a power series in a given function of $t$ is suggested in [76]. The methods which are extensions of this power series expansion formalism to a general Fokker-Planck-Schrödinger process are presented in [77]. They are applied to a well-known problem of the decay of an unstable state driven by exponentially correlated Gaussian noise.

\section{B. Regime of purely in-plane reorientation: 2D model}

In Sec. III A our model has been formulated as the free energy FP equation [Eq. (43)] describing rotational diffusion of azo-dye molecules governed by effective mean-field potential (51). Since general analysis can be rather involved, we first examine our model in the limiting two-dimensional case of purely in-plane reorientation.

The simplified two-dimensional model,

$$
\begin{aligned}
\partial_{\tau} f & =\partial_{\phi}\left[\partial_{\phi} f+f \partial_{\phi} V\right] \\
& =\partial_{\phi}^{2} f+\frac{1}{2}\left[\partial_{\phi}^{2}(f V)+f \partial_{\phi}^{2} V-V \partial_{\phi}^{2} f\right], \quad \tau=D_{z}^{(\mathrm{rot})} t,
\end{aligned}
$$

can be derived by assuming that the out-of-plane component of the unit vector $\hat{\mathbf{u}}$ describing orientation of the azo-dye molecules is suppressed and, as is shown in Fig. 1, $\hat{\mathbf{u}}$ $=(\sin \phi, \cos \phi, 0)$. It implies that, similar to the model of a single axis rotator with two equivalent sites [56,57], the molecules are constrained to be parallel to the substrate plane (the $x-y$ plane) and the orientational distribution function takes the factorized form

$$
f(\hat{\mathbf{u}}, t)=f(\phi, t) \delta(c), \quad c=\cos \theta,
$$

where $\delta(c)$ is the $\delta$ function.

So, in this section we consider the FP equation [Eq. (55)] representing the simplest case when the out-of-plane reorientation has been completely suppressed. Then, for $\hat{\mathbf{u}}$ $=(\sin \phi, \cos \phi, 0)$, the diagonal elements of order parameter tensor (12) averaged over the azimuthal angle are given by

$$
S_{x}=\left\langle Q_{x x}\right\rangle=\frac{1}{2}\left\langle 3 \sin ^{2} \phi-1\right\rangle=\frac{1}{4}[-3\langle\cos 2 \phi\rangle+1],
$$

$$
S_{y}=\left\langle Q_{y y}\right\rangle=\frac{1}{2}\left\langle 3 \cos ^{2} \phi-1\right\rangle=\frac{1}{4}[3\langle\cos 2 \phi\rangle+1],
$$

$$
S_{z}=\left\langle Q_{z z}\right\rangle=-\frac{1}{2},
$$

where $\langle\cdots\rangle=\int_{0}^{2 \pi} \cdots d \phi$.

We can now substitute order parameters (57) into the effective potential $V$ given in Eq. (51). The result for the angular dependent part of the potential is

$$
V=\left(v_{1}+v_{2}\langle\cos 2 \phi\rangle\right) \cos 2 \phi \equiv v \cos 2 \phi,
$$

where $v_{1} \equiv 3 u_{I} I_{\mathrm{UV}} / 4$ and $v_{2}=9 u_{2} / 8$ are the photoexcitation and intermolecular interaction parameters, respectively.

Our next step is to obtain the system of equation for the averaged harmonics, $c_{n}(\tau)=\langle\cos n \phi\rangle(\tau)$, which are proportional to the Fourier coefficients of the distribution function, $f(\phi, \tau)$. To this end, we integrate the FP equation [Eq. (55)] multiplied by $\cos n \phi$ over the azimuthal angle. The resulting system reads

$$
\partial_{\tau} c_{n}=-n^{2} c_{n}+n v\left(c_{n+2}-c_{|n-2|}\right), \quad c_{0}=1,
$$

where $v=v_{1}+v_{2} c_{2}$.

When $f(\phi+\pi)=f(\phi)$, the odd numbered harmonics vanish, $c_{2 k+1}=0$. For the even numbered harmonics, $p_{k} \equiv c_{2 k}$, system (59) can be conveniently recast into the form

$$
\begin{gathered}
\partial_{\tau} p_{k}=-4 k^{2} p_{k}+2 k v\left(p_{k+1}-p_{k-1}\right), \quad k=1,2, \ldots, \\
p_{k} \equiv c_{2 k}, \quad p_{0}=c_{0}=1, \quad v=v_{1}+v_{2} p_{1},
\end{gathered}
$$

where $p_{1}=\langle\cos 2 \phi\rangle$ is the order parameter harmonics that enter the expressions for orientational order parameters (57).

\section{Bifurcations of stationary states}

From the general formula [Eq. (52)] we obtain the expression for the stationary distributions,

$$
\begin{gathered}
f_{\mathrm{st}}=Z_{\mathrm{st}}^{-1} \exp [-V]=Z_{\mathrm{st}}^{-1} \exp [-v \cos 2 \phi], \\
Z_{\mathrm{st}}=\int_{0}^{2 \pi} \exp [-V] d \phi,
\end{gathered}
$$

representing the photosteady states in the two-dimensional case with potential (58). 
Equation (62) can now be combined with the relation

$$
\exp [-v \cos 2 \phi]=I_{0}(v)+2 \sum_{k=1}^{\infty}(-1)^{k} I_{k}(v) \cos 2 k \phi,
$$

where $I_{k}$ is the modified Bessel function of integer order [78], to derive the formulas

$$
\begin{gathered}
Z_{\mathrm{st}}=2 \pi I_{0}(v), \\
p_{k}^{\text {(st) }}=(-1)^{k} I_{k}(v) / I_{0}(v),
\end{gathered}
$$

giving the stationary state statistical integral, $Z_{\mathrm{st}}$, and the averaged harmonics, $p_{k}^{(\text {st) }}$, expressed in terms of the parameter $v$. Using the recurrence relation [78]

$$
v\left[I_{k-1}(v)-I_{k+1}(v)\right]=2 k I_{k}(v),
$$

it is not difficult to verify that formula (65) gives the stationary solution to system (60) which, in the steady state regime with $\partial_{\tau} p_{k}=0$, is represented by the finite-difference equation

$$
v\left(p_{k+1}^{(\mathrm{st})}-p_{k-1}^{(\mathrm{st})}\right)=2 k p_{k}^{(\mathrm{st})} .
$$

From Eq. (58), the parameter $v=v_{1}+v_{2} p_{1}^{(\text {st) }}$ depends on the order parameter harmonics and Eq. (65) with $k=1$ provides the self-consistency condition

$$
p_{1}^{(\mathrm{st})}=\left(v-v_{1}\right) / v_{2}=-I_{1}(v) / I_{0}(v) .
$$

This condition can also be obtained as the stationary point equation for stationary state free energy (54). In our case, we have

$$
\begin{gathered}
U\left[f_{\mathrm{st}}\right]=v p_{1}^{(\mathrm{st})}-\frac{v_{2}}{2}\left[p_{1}^{(\mathrm{st})}\right]^{2}, \\
\left\langle\ln f_{\mathrm{st}}\right\rangle=-\ln Z_{\mathrm{st}}-v p_{1}^{(\mathrm{st})}, \\
F_{\mathrm{st}}(v)=v_{2}^{-1}\left[-\frac{v^{2}}{2}+v_{1} v\right]-\ln I_{0}(v),
\end{gathered}
$$

where the additive constant is chosen so as to have the free energy vanishing at $v=0$.

In Fig. 2(a) it is illustrated that, in the $v-p_{1}$ plane, solutions of the self-consistency [Eq. (68)] can be found as intersection points of the curve, $-I_{1}(v) / I_{0}(v)$, and the straight line, $\left(v-v_{1}\right) / v_{2}$. It is seen that the number of the intersection points varies between one and three depending on the values of the parameters $v_{1}$ and $v_{2}$.

As is shown in Fig. 2(b), for the case of three stationary states, the free energy curves are of the double-well potential form with two local minima separated by the energy barrier. From the lowest order term of the series expansion,

$$
-I_{1}(x) / I_{0}(x) \approx-\frac{x}{2}+\frac{x^{3}}{16}-\frac{x^{5}}{97},
$$

it is not difficult to see that this case may occur only if the parameter $v_{2}$ is less than -2 .

Referring to Fig. 2(b), at $v_{1}=0$ and $v_{2}<-2$, free energy (71) is an even function of $v, F_{\mathrm{st}}(v)=F_{\mathrm{st}}(-v)$, with two sym-
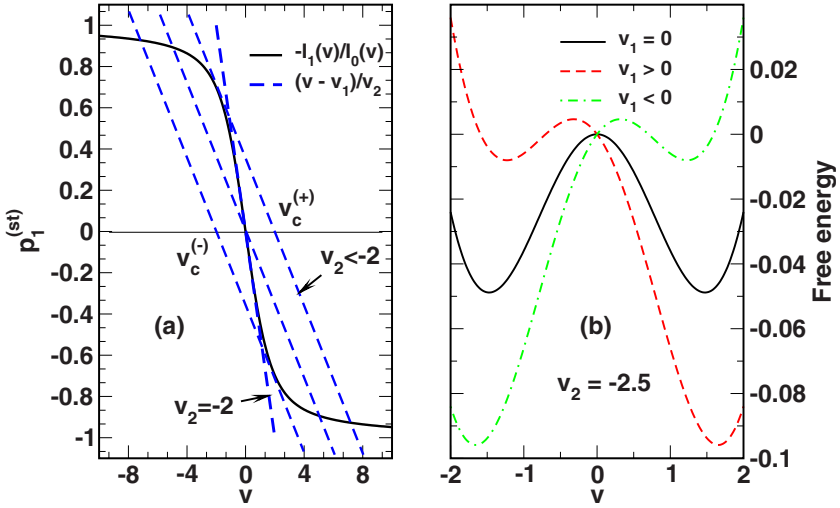

FIG. 2. (Color online) (a) Intersection points represent solutions of self-consistency equation (68). (b) Stationary state free energy (54) as a function of the parameter $v$.

metrically arranged minima representing two stable stationary states. When the parameter of intermolecular interaction, $v_{2}$, increases passing through its critical value, $v_{2}^{(c)}=-2$, the minima come close together and coalesce at the critical point. So, at $v_{2}>-2$, there is only one minimum corresponding to the unique equilibrium state.

When the activating light is switched on, the parameter $v_{1}$ is distinct from zero. It gives rise to asymmetry effects illustrated in Fig. 2(b). It can be seen that, at $v_{1} \neq 0$, one of two minima becomes metastable. The local maximum representing the unstable stationary state and the metastable minima merge and disappear provided the magnitude of the parameter $v_{1}$ is sufficiently large.

This effect is evident from the curve depicted in Fig. 3(a) where the stationary state order parameter harmonics is plotted in the $v_{1}-p_{1}$ plane by using the following parametrization:

$$
p_{1}^{(\mathrm{st})}=\left\{\begin{array}{l}
p_{1}=p_{1}(\xi)=-I_{1}(\xi) / I_{0}(\xi) \\
v_{1}=v_{1}(\xi)=\xi-v_{2} p_{1}(\xi) .
\end{array}\right.
$$

So, the free energy has two local minima only if the inequalities
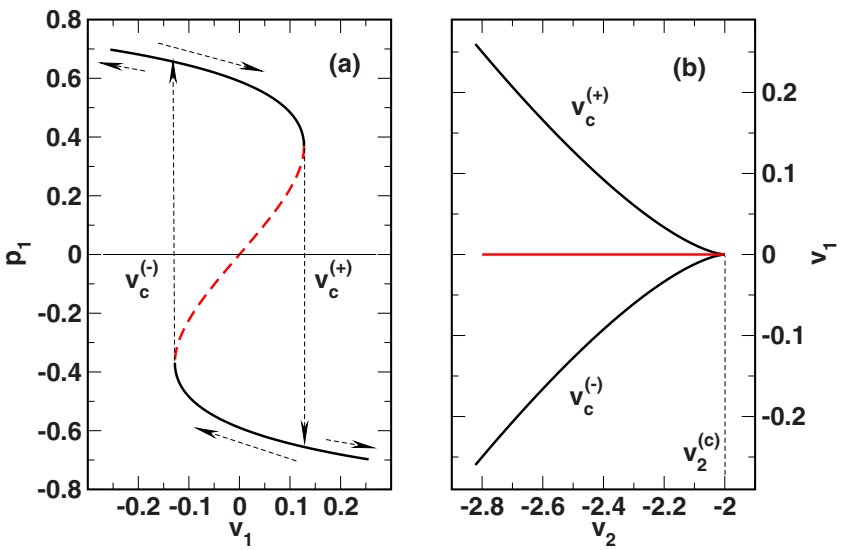

FIG. 3. (Color online) (a) Order parameter harmonics $p_{1}$ $=\langle\cos 2 \phi\rangle$ as a function of the photoexcitation parameter $v_{1}$ at $v_{2}$ $=-2.5$; (b) bifurcation curves in the $v_{2}-v_{1}$ plane are typical of the cusp catastrophe with the cusp singularity located at $(-2,0)$. 


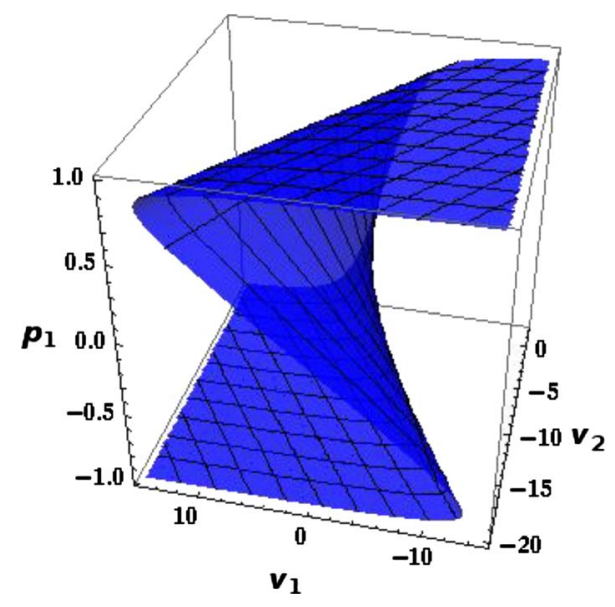

FIG. 4. (Color online) Bifurcation diagram as the cusp surface in the three-dimensional $\left(v_{1}, v_{2}, p_{1}\right)$ space.

$$
v_{2}<v_{2}^{(c)}=-2, \quad v_{c}^{(-)}<v_{1}<v_{c}^{(+)}
$$

are satisfied. The critical values of the parameter $v_{1}$ depend on the reduced strength of intermolecular interaction $v_{2}$ and can be parametrized as follows:

$v_{c}=\left\{\begin{array}{l}v_{1}=v_{1}(\xi)=\xi-v_{2} p_{1}(\xi) \\ v_{2}=v_{2}(\xi)=-\left\{\left[I_{1}(\xi) / I_{0}(\xi)\right]_{\xi}^{\prime}\right\}^{-1}=\frac{1}{1+p_{1}(\xi) / \xi-p_{1}^{2}(\xi)} .\end{array}\right.$

Geometrically, in the $v_{2}-v_{1}$ plane, Eq. (75) defines the bifurcation curves shown in Fig. 3(b). These curves form a bifurcation set which is the projection of the cusp surface,

$$
S_{B}=\left\{\begin{array}{l}
v_{1}=\xi-\zeta p_{1}(\xi) \\
v_{2}=\zeta \\
p_{1}=-I_{1}(\xi) / I_{0}(\xi)
\end{array}\right.
$$

representing the bifurcation diagram in the three-dimensional $\left(v_{1}, v_{2}, p_{1}\right)$ space (see Fig. 4). Note that the cusp bifurcation occurs as a canonical model of a codimension 2 singularity [79] and the surface shown in Fig. 4 is typical of the cusp catastrophe $[80,81]$.

We conclude this section with the remark on how diffusion models may account for the effect of long-term stability by using approximation of the "frozen" potential proposed in Ref. [43]. Mathematically, it implies that, after switching off the exciting light at time $t=t_{\text {off }}$ with the order parameter harmonics $p_{\text {off }}=p_{1}\left(t_{\text {off }}\right)$, the relaxation process is governed by the kinetic equations for harmonics (60) where the parameter $v$ is changed to the frozen interaction parameter $v_{\text {off }}=v_{2} p_{\text {off }}$. From Eq. (65) the stationary value of the order parameter harmonics

$$
p_{\text {off }}^{\text {(st) }}=-I_{1}\left(v_{\text {off }}\right) / I_{0}\left(v_{\text {off }}\right) \equiv p_{1}\left(v_{\text {off }}\right)
$$

is determined by the frozen strength of intermolecular interaction, $v_{\text {off }}$, and thus depends on the value of the order parameter harmonics at the time of switching, $t=t_{\text {off }}$. So, in the two-state and in the 2D diffusion models the memory effect underlying the long-term stability of POA is described by relations (34) and (77), respectively.

\section{RESULTS}

In Secs. II C and III B, we have employed the approaches based on the master and Fokker-Planck equations to introduce two different models: the two-state model and the twodimensional diffusion model, respectively. In both cases, the photoinduced anisotropy is characterized by the orientational order parameters whose temporal evolution is governed by the kinetic equations of the model.

In Sec. II B, we discussed how the order parameters can be related to absorption characteristics such as extinction (absorption) coefficients and optical densities. Specifically, Eq. (22) shows that the absorption order parameters, $S_{i}^{(a)}$, defined in Eq. (17) as a function of the principal values of the extinction coefficients, $\kappa_{i}$, are proportional to the orientational order parameters [Eq. (14)]. A comparison between the theory and experiment can thus be made from the measured values of the absorption coefficients.

In thin anisotropic films, the absorption coefficients can be determined experimentally using the methods of ellipsometry $[82,83]$. These are generally based on the analysis of the polarization state of light reflected from or transmitted through a sample.

One of the simplest experimental procedures is to measure the light transmittance of a film when the testing beam is normally incident and linearly polarized. Performing the measurements for beams polarized perpendicular and parallel to the polarization vector of the UV light the two in-plane optical densities, $D_{y}^{(a)}=D_{\|}^{(a)}$ and $D_{x}^{(a)}=D_{\perp}^{(a)}$, can be obtained as a function of the irradiation dose.

The normal component, $D_{z}^{(a)}$, then can be estimated by assuming that the total sum of principal optical densities

$$
D_{\text {tot }}^{(a)}=D_{x}^{(a)}+D_{y}^{(a)}+D_{z}^{(a)}
$$

does not depend on the irradiation dose and the photosaturated state is uniaxially anisotropic with $D_{y}^{(a)}=D_{z}^{(a)}$. More details about this approach can be found, e.g., in Refs. $[35,40]$ where it was applied to azopolymer films.

In the Appendix we show that the absorption extinction coefficients can be extracted from the dependence of absorbance on the incidence angle measured using probe beams which are linearly polarized parallel ( $p$ polarization) and perpendicular ( $s$ polarization) to the plane of incidence. In order to fit the experimentally measured curves, this method relies on the analytical expressions for the transmission coefficients of biaxially anisotropic absorbing layers deduced in the Appendix [see Eq. (A22)].

The results for the extinction coefficients, $\kappa_{x}, \kappa_{y}$, and $\kappa_{z}$, are summarized in Fig. 5 where the coefficients are plotted against the irradiation time. The corresponding absorption order parameters, $S_{x}^{(a)}, S_{y}^{(a)}$, and $S_{z}^{(a)}$, evaluated from the experimental data by using formula (17), are presented in Fig. 6.

It can be seen that the initial and photosaturated states are both uniaxially anisotropic with $S_{x}^{(a)}=S_{y}^{(a)}$ and $S_{z}^{(a)}=S_{y}^{(a)}$, respectively. So, similar to the case of azopolymers, the tran- 


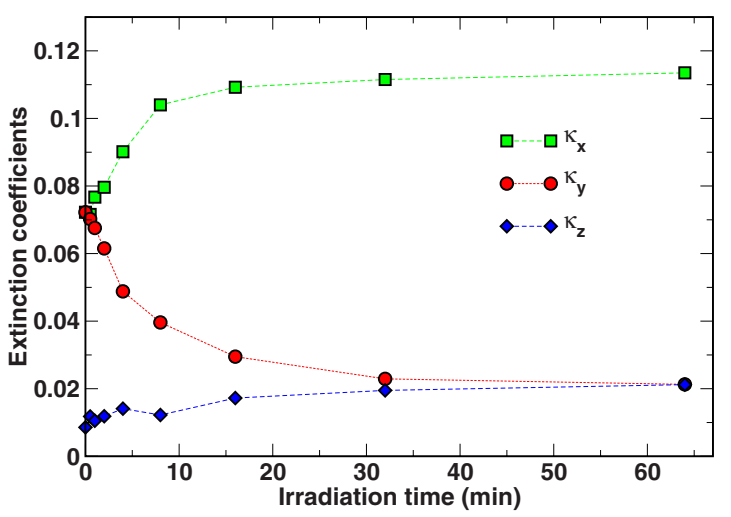

FIG. 5. (Color online) Extinction coefficients as a function of irradiation time.

sient photoinduced orientational structures are inevitably biaxial.

It is also clear that, before reaching the regime of photosaturation, the in-plane order parameters, $S_{x}^{(a)}$ and $S_{y}^{(a)}$, undergo pronounced changes. By contrast, the normal component of the order parameter, $S_{z}^{(a)}$, slowly increases with irradiation time. We can therefore employ the twodimensional diffusion model described in Sec. III B as a zero order approximation where variations in the normal order parameter component, $S_{z}^{(a)}$, are neglected.

The theoretical curves shown in Fig. 6 as dashed lines are computed by solving system (60). The fitting procedure is as follows.

Assuming that the order parameter $S_{z}^{(a)}$ is constant and $S_{z}^{(a)} \approx-0.334$, we obtain the coefficient $r_{a}$ that enters relation $(22), r_{a} \approx 0.67$, and the absorption anisotropy parameter $\sigma_{a}$ $\approx 6.1$. Then, from the experimental data, we can estimate the order parameter harmonics in the photosteady state, $p_{1}^{(\mathrm{st})} \approx$ -0.887 (the corresponding in-plane order parameter is $S_{x x}^{(\mathrm{st})}$ $\approx 0.9$ ). The self-consistency condition [Eq. (68)] gives the equation $v_{2}=v_{\text {off }}^{\text {(st) }} / p_{1}\left(v_{\text {off }}^{\text {(st) }}+v_{1}\right)$, linking the interaction and the photoexcitation parameters, where $v_{\text {off }}^{\text {(st) }}$ is the solution of Eq. (77) at $p_{\mathrm{off}}^{(\mathrm{st})}=p_{1}^{(\mathrm{st})}: p_{1}^{(\mathrm{st})}=p_{1}\left(v_{\mathrm{off}}^{(\mathrm{st})}\right)$. At $v_{1}=1.0$, this equation yields the value of the intermolecular interaction parameter, $v_{2} \approx-5.22$.

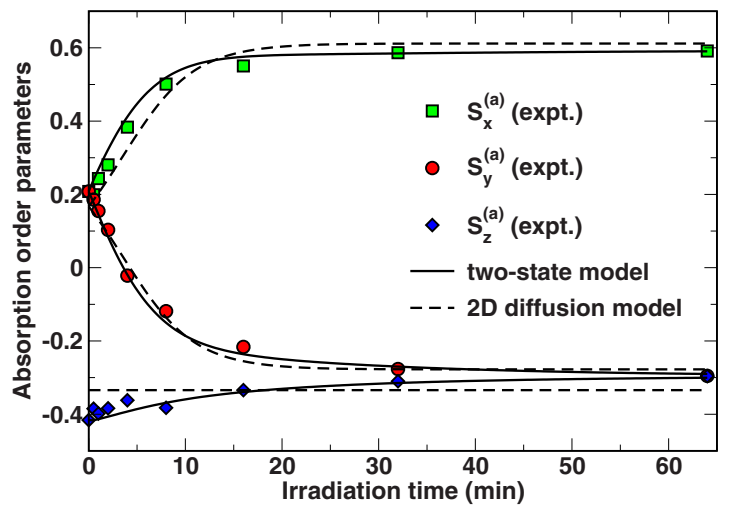

FIG. 6. (Color online) Absorption order parameters as a function of irradiation time. The theoretical curves are computed by numerically solving the kinetic equations for two-state model (27) and for two-dimensional diffusion model (60).
According to the experimental data presented in Figs. 5 and 6 , the irradiation time it takes to reach the regime of photosaturation is about $64 \mathrm{~min}$. From the other hand, for the computed dependence of the order parameter harmonics, $p_{1}$, on $\tau \equiv D_{z}^{(\text {rot })} t$, this regime takes place at $\tau \geq 1.1$. So, the rotational diffusion constant $D_{z}^{(\text {rot })}$ can be estimated at about $0.013 \mathrm{~min}^{-1}\left(\approx 2.2 \times 10^{-4} \mathrm{~s}^{-1}\right)$.

Referring to Fig. 6, agreement between the theoretical curves and experiment indicates that the two-dimensional diffusion model can be regarded as a good approximation to start from. So, the regime of kinetics of the photoinduced structures in the azo-dye film appears to be close to the limiting case of the in-plane reorientation.

Now we consider the two-state model formulated in Sec. II C. Similar to the case of the 2D diffusion model, our first step is to determine the coefficient $r_{a}$ and the anisotropy parameter $\sigma_{a}$. The coefficient $r_{a}, r_{a} \approx 0.89$, can be calculated as the solution of the equation obtained by substituting the photosaturated value of the absorption order parameter, $S_{x x}^{(a)}$ $=r_{a} S_{\mathrm{st}} \approx 0.59$, into Eq. (38). We also find that the absorption anisotropy parameter $\sigma_{a}$ is about $25.1, \sigma_{a} \approx 25.1$, and the photosteady state is characterized by the order parameter $S_{\mathrm{st}} \equiv S_{x x}^{(\mathrm{st})} \approx 0.66$.

The numerical results shown in Fig. 6 are computed in the regime of photoreorientation where the decay rate of the excited state $\gamma_{e} \approx 11.3 \mathrm{~min}^{-1}$ is much larger than the excitation rate, $\gamma_{p h} / \gamma_{e} \approx 2 \times 10^{-3}$, and the thermal relaxation rates, $\gamma_{\mathrm{G}} / \gamma_{e} \approx 2 \times 10^{-3}$ and $\gamma_{m} / \gamma_{e} \approx 8 \times 10^{-3}$. Numerical calculations in the presence of irradiation were followed by computing the stationary values of $S$ and $\Delta S$ to which the order parameters decay after switching off the irradiation at time $t_{\text {off }}$ (for more details see Sec. II C).

The results presented in Fig. 6 suggest that the two-state model can be used to relax the assumption on purely in-plane photoreorientation and to go beyond the limitations of the 2D diffusion model.

\section{DISCUSSION AND CONCLUSIONS}

In order to study the kinetics of the photoinduced ordering in azo-dye films we have employed two different models capable to account for the peculiarities of the process. In particular, the films under consideration exhibit the longterm stable POA characterized by the weak photochromism and by the suppressed out-of-plane photoreorientation leading to the biaxiality effects.

The two-state model is formulated by using the phenomenological approach developed in Refs. [35,40]. In this approach, the film is represented by an ensemble of two-level molecular systems. So, it starts from the master equation [Eq. (1)] for one-particle angular distribution functions of the ground-state and excited molecules. The kinetics is then determined by the angular redistribution probabilities that enter the photoexcitation and decay rates. They also define coupling between the azo-dye molecules and the anisotropic field represented by the distribution function of the matrix $f_{m}$. This anisotropic field reflects the presence of long-living angular correlations and stabilizes the photoinduced anisotropy. 
The resulting kinetic equations [Eq. (27)] for the order parameter components are deduced by using the parabolic approximation suggested in Ref. [35] to express the order parameter correlation functions in terms of the order parameter tensor. Following the procedure described in Refs. $[23,35]$, these order parameter correlation functions are additionally modified in order to take into account constraints suppressing out-of-plane reorientation. Another important assumption taken in our two-state model is that the excited molecules do not affect the ordering kinetics directly.

Similarly, there is an alternative approach which is formulated in Sec. III without explicit reference to excited electronic levels. According to this approach, the photoinduced anisotropy arises from the rotational Brownian motion of azo-dye molecules in the effective light modified potential. Mathematically, this suggests using the mean-field FokkerPlanck equation [Eq. (40)] with effective free energy functional (41) as the equations governing the kinetics of photoinduced ordering. Thus diffusion models can be defined by specifying the rotational diffusion tensor and effective potential (42) that enter Eq. (43).

The two-dimensional model studied in Sec. III B presents the simplest case to start from. It is based on the approximation of purely in-plane photoreorientation which assumes that the normal order parameter component $S_{z}$ was kept constant.

In order to test applicability of this approximation, we compared the predictions of this simple model with the available experimental data. From Fig. 6 it is clear that, in azodye films, the kinetics of photoinduced structures take place in the regime close to the limiting case of purely in-plane photoreorientation.

Referring to Fig. 6, the comparison between the numerical results and the experimental data shows that the two-state and the 2D diffusion models both correctly capture the basic features of the photo-ordering kinetics in the azo-dye layers. It comes as no surprise that the results computed from the two-level model give better agreement with experiment than the ones for the 2D diffusion model. The primary reason for this is that the two-state model takes into account effects due to variations in $S_{z}$.

These effects can also be taken into consideration in the rotational diffusion approach by expanding the orientational distribution function into a series over the spherical harmonics, $Y_{l m}(\hat{\mathbf{u}})$, or, more generally, the Wigner $D$ functions $[53,84], D_{m m^{\prime}}^{j}(\boldsymbol{\omega})$. The mean-field Fokker-Planck equation [Eq. (43)] then can be transformed into the system of nonlinear ordinary differential equations for the averaged harmonics, $\left\langle Y_{l m}\right\rangle(t)$ or $\left\langle D_{m m^{\prime}}^{j}\right\rangle(t)$. Equations (60) represent the special case of such system derived for the two-dimensional model.

For an infinite number of equations, numerical analysis involves truncating the system so that only a finite number of harmonics are taken into account. The number of harmonics is typically determined by the required accuracy of calculations. Difficulties emerge if this number turns out to be very large. For instance, this is the case for highly ordered photosteady states.

So, we have demonstrated that the phenomenological approach in Ref. [35] and generalized diffusion models can be

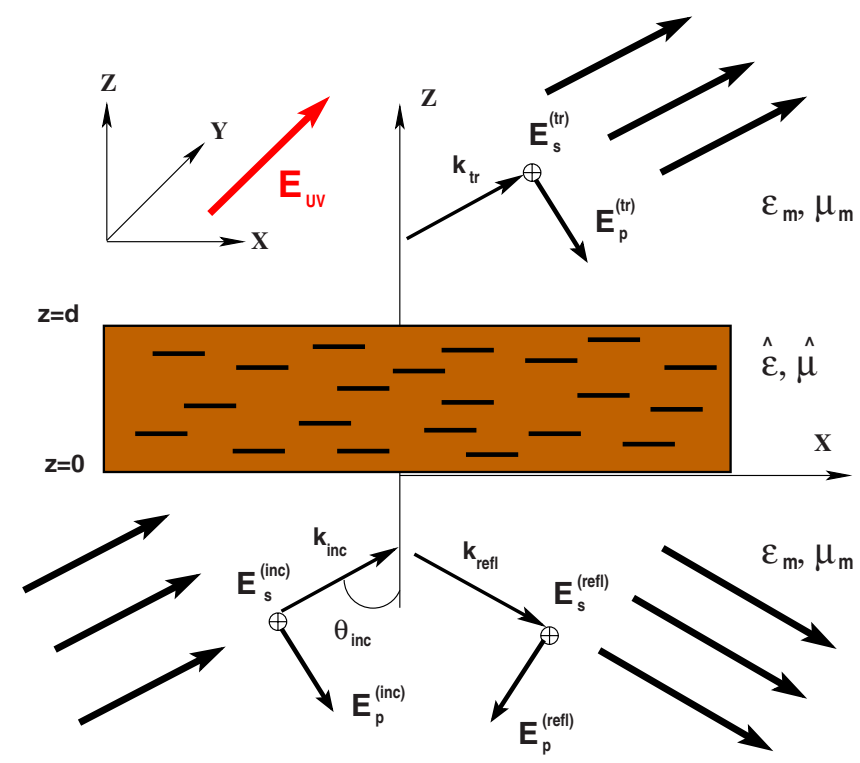

FIG. 7. (Color online) Geometry of anisotropic layer in the plane of incidence.

used as useful tools for studying photoinduced ordering processes in azo-dye films. It should be noted, however, that theoretical approaches of this sort, by definition, do not involve explicit considerations of microscopic details of azodye film physics. A more comprehensive study is required to relate the effective parameters of the models and physical parameters characterizing interactions between molecular units of films.

\section{ACKNOWLEDGMENTS}

This work was supported by HKUST CERG Grants No. 612208, No. 612406, and No. RPC07/08.EG01 and RGC Grant No. 614807. We thank A. Murauski and A. Muravsky for stimulating remarks and help with the input data used in Appendix. A.D.K. is also grateful to Professor B. I. Lev (Bogoliubov Institute of Theoretical Physics, Kiev) for useful discussions and acknowledges partial financial support from STCU Grant No. 4687.

\section{APPENDIX: TRANSMISSION COEFFICIENTS OF BIAXIALLY ANISOTROPIC ABSORBING LAYERS}

In this section we derive the exact solution to the transmission boundary value problem by applying the theoretical approach developed in Refs. $[85,86]$ to the case of biaxially (and uniformly) anisotropic absorbing layers.

As is shown in Fig. 7, we consider an absorbing uniformly anisotropic film of thickness $d$ with the $z$ axis giving the optic axis normal to the bounding surfaces: $z=0$ and $z$ $=d$. The other two in-plane optic axes are assumed to be directed along the unit vectors $\hat{\mathbf{x}}$ and $\hat{\mathbf{y}}$. In this case the dielectric tensor of the film is diagonal and is defined in Eq. (15). From Eq. (16), the principal values of the tensor, $\epsilon_{\alpha}$, can be expressed in terms of the refractive indices, $n_{\alpha}^{(r)}$, and the extinction coefficients, $\kappa_{\alpha}$. 
The medium surrounding the layer is assumed to be optically isotropic and characterized by the dielectric constant $\epsilon_{m}$ and the magnetic permittivity $\mu_{m}$. Referring to Fig. 7, there are two plane waves in the half space $z \leq 0$ bounded by the entrance face of the layer: the incoming incident wave $\left\{\mathbf{E}_{\text {inc }}, \mathbf{H}_{\text {inc }}\right\}$ and the outgoing reflected wave $\left\{\mathbf{E}_{\text {refl }}, \mathbf{H}_{\text {refl }}\right\}$. In the half space $z \geq d$ after the exit face, the only wave is the transmitted plane wave $\left\{\mathbf{E}_{\text {trans }}, \mathbf{H}_{\text {trans }}\right\}$ which propagates along the direction of incidence and is excited by the incident light.

So, the electric field outside the layer is a superposition of the plane waves

$$
\begin{gathered}
\left.\mathbf{E}\right|_{z<0}=\mathbf{E}_{\text {inc }}\left(\hat{\mathbf{k}}_{\text {inc }}\right) e^{i\left(\mathbf{k}_{\text {inc }} \cdot \mathbf{r}\right)}+\mathbf{E}_{\text {refl }}\left(\hat{\mathbf{k}}_{\text {refl }}\right) e^{i\left(\mathbf{k}_{\text {refl }} \cdot \mathbf{r}\right)}, \\
\left.\mathbf{E}\right|_{z>d}=\mathbf{E}_{\text {tr }}\left(\hat{\mathbf{k}}_{\text {tr }}\right) e^{i\left(\mathbf{k}_{\text {trr }} \cdot \mathbf{r}\right)},
\end{gathered}
$$

where the wave vectors $\mathbf{k}_{\text {inc }}, \mathbf{k}_{\text {refl }}$, and $\mathbf{k}_{\text {tr }}$ are constrained to lie in the plane of incidence due to the boundary conditions requiring the tangential components of the electric and magnetic fields to be continuous at the boundary surfaces.

Another consequence of the boundary conditions is that the tangential components of the wave vectors are the same. Assuming that the incidence plane is the $x-z$ plane we have

$$
\mathbf{k}_{\alpha}=k_{\mathrm{vac}} \mathbf{q}_{\alpha}=k_{m} \hat{\mathbf{k}}_{\alpha}=k_{x} \hat{\mathbf{x}}+k_{z}^{(\alpha)} \hat{\mathbf{z}}, \quad \alpha \in\{\text { inc, } \operatorname{refl}, \operatorname{tr}\},
$$

where $k_{m} / k_{\mathrm{vac}}=n_{m}=\sqrt{\mu_{m} \epsilon_{m}}$ is the refractive index of the ambient medium and $k_{\mathrm{vac}}=\omega / \mathrm{c}$ is the free-space wave number. The wave vector components can now be expressed in terms of the incidence angle $\theta_{\text {inc }}$ as follows:

$$
\begin{gathered}
k_{x}=k_{m} \sin \theta_{\mathrm{inc}} \equiv k_{\mathrm{vac}} q_{x}, \\
k_{z}^{(\mathrm{inc})}=k_{z}^{(\mathrm{rr})}=-k_{z}^{(\mathrm{refl})}=k_{m} \cos \theta_{\mathrm{inc}} \equiv k_{\mathrm{vac}} q_{m}, \\
q_{x}=n_{m} \sin \theta_{\mathrm{inc}}, \quad q_{m}=\sqrt{n_{m}^{2}-q_{x}^{2}} .
\end{gathered}
$$

The plane wave traveling in the isotropic ambient medium along the wave vector [Eq. (A2)] is transverse, so that the polarization vector is given by

$$
\mathbf{E}_{\alpha}\left(\hat{\mathbf{k}}_{\alpha}\right)=E_{p}^{(\alpha)}\left(k_{z}^{(\alpha)} \hat{\mathbf{x}}-k_{x} \hat{\mathbf{z}}\right) k_{m}^{-1}+E_{s}^{(\alpha)} \hat{\mathbf{y}},
$$

where $E_{p}^{(\alpha)}$ and $E_{s}^{(\alpha)}$ are the in-plane and out-of-plane components of the electric field, respectively.

The electromagnetic field of incident, transmitted, and reflected waves propagating in the ambient medium is of the general form

$$
\{\mathbf{E}, \mathbf{H}\}=\{\mathbf{E}(z), \mathbf{H}(z)\} e^{i\left(k_{x} x-\omega t\right)} .
$$

On substituting the relations [Eq. (A7)] into the Maxwell equations we can obtain the equations for the tangential components of the electromagnetic field inside the anisotropic layer. The result can be written in the following $4 \times 4$ matrix form:

$$
-i \partial_{\tau} \mathbf{F}=\mathbf{M} \cdot \mathbf{F} \equiv\left(\begin{array}{ll}
\mathbf{M}_{11} & \mathbf{M}_{12} \\
\mathbf{M}_{21} & \mathbf{M}_{22}
\end{array}\right)\left(\begin{array}{l}
\mathbf{E}_{P} \\
\mathbf{H}_{P}
\end{array}\right), \quad \tau \equiv k_{\mathrm{vac}} z
$$

where $\mathbf{E}_{P}(z)=\left(\begin{array}{c}E_{x}(z) \\ E_{y}(z)\end{array}\right)$ and $\mathbf{H}_{P}(z)=\left(\begin{array}{c}H_{y}(z) \\ -H_{\mathrm{r}}(z)\end{array}\right)$.

For dielectric tensor (15) with the plane of incidence parallel to the $x-z$ plane, from the general expressions derived in Refs. [85,86], the $2 \times 2$ matrices $\mathbf{M}_{i j}$ characterizing the block structure of the matrix $\mathbf{M}$ are given by

$$
\begin{array}{ll}
\mathbf{M}_{12}=\epsilon_{z}^{-1}\left(\begin{array}{cc}
n_{z}^{2}-q_{x}^{2} & 0 \\
0 & n_{z}^{2}
\end{array}\right), & \mathbf{M}_{11}=\mathbf{0}, \\
\mathbf{M}_{21}=\mu^{-1}\left(\begin{array}{cc}
n_{x}^{2} & 0 \\
0 & n_{y}^{2}-q_{x}^{2}
\end{array}\right), \quad \mathbf{M}_{22}=\mathbf{0} .
\end{array}
$$

According to the computational procedure developed in Refs. $[85,86]$, the transmission and reflection matrices defined through the linear input-output relations

$$
\left(\begin{array}{c}
E_{p}^{(\mathrm{tr})} \\
E_{s}^{\text {(tr) }}
\end{array}\right)=\mathbf{T}\left(\begin{array}{c}
E_{p}^{(\mathrm{inc})} \\
E_{s}^{\text {(inc) }}
\end{array}\right), \quad\left(\begin{array}{c}
E_{p}^{(\mathrm{refl})} \\
E_{s}^{(\mathrm{refl})}
\end{array}\right)=\mathbf{R}\left(\begin{array}{c}
E_{p}^{(\mathrm{inc})} \\
E_{s}^{\text {(inc) }}
\end{array}\right)
$$

can be expressed in terms of the linking matrix

$$
\mathbf{W}=\mathbf{V}_{m}^{-1} \cdot \mathbf{U}^{-1}(h) \cdot \mathbf{V}_{m}=\left(\begin{array}{ll}
\mathbf{W}_{11} & \mathbf{W}_{12} \\
\mathbf{W}_{21} & \mathbf{W}_{22}
\end{array}\right)
$$

as follows:

$$
\begin{gathered}
\mathbf{T}=\mathbf{W}_{11}^{-1}, \\
\mathbf{R}=\mathbf{W}_{21} \cdot \mathbf{W}_{11}^{-1}=\mathbf{W}_{21} \cdot \mathbf{T} .
\end{gathered}
$$

The expression for linking matrix (A12) involves the inverse of the evolution operator

$$
\mathbf{U}^{-1}(h)=\mathbf{U}(-h)=\exp \{-i \mathbf{M} h\}, \quad h=k_{\mathrm{vac}} d
$$

and the eigenvector matrix for the ambient medium

$$
\mathbf{V}_{m}=\left(\begin{array}{cc}
\mathbf{E}_{m} & -\boldsymbol{\sigma}_{3} \mathbf{E}_{m} \\
\mathbf{H}_{m} & \boldsymbol{\sigma}_{3} \mathbf{H}_{m}
\end{array}\right),
$$

which is characterized by the two diagonal $2 \times 2$ matrices, $\mathbf{E}_{m}=\operatorname{diag}\left(q_{m} / n_{m}, 1\right)$ and $\mu_{m} \mathbf{H}_{m}=\operatorname{diag}\left(n_{m}, q_{m}\right)$, where $\boldsymbol{\sigma}_{3}$ $=\operatorname{diag}(1,-1)$.

In our case, the resulting expression for the evolution operator is

$$
\begin{aligned}
\mathbf{U}(h)=\exp \{i \mathbf{M} h\} & =\mathbf{V} \cdot\left(\begin{array}{cc}
\mathbf{U}_{+} & \mathbf{0} \\
\mathbf{0} & \mathbf{U}_{-}
\end{array}\right) \mathbf{N}^{-1} \cdot \mathbf{V}^{T} \cdot \mathbf{G}, \\
\mathbf{G} & =\left(\begin{array}{cc}
\mathbf{0} & \mathbf{I}_{2} \\
\mathbf{I}_{2} & \mathbf{0}
\end{array}\right),
\end{aligned}
$$

where $\mathbf{I}_{2}=\operatorname{diag}(1,1)$,

$$
\mathbf{U}_{ \pm}=\exp \{ \pm i \mathbf{Q} h\}, \quad \mathbf{Q}=\left(\begin{array}{cc}
q_{p} & 0 \\
0 & q_{s}
\end{array}\right),
$$



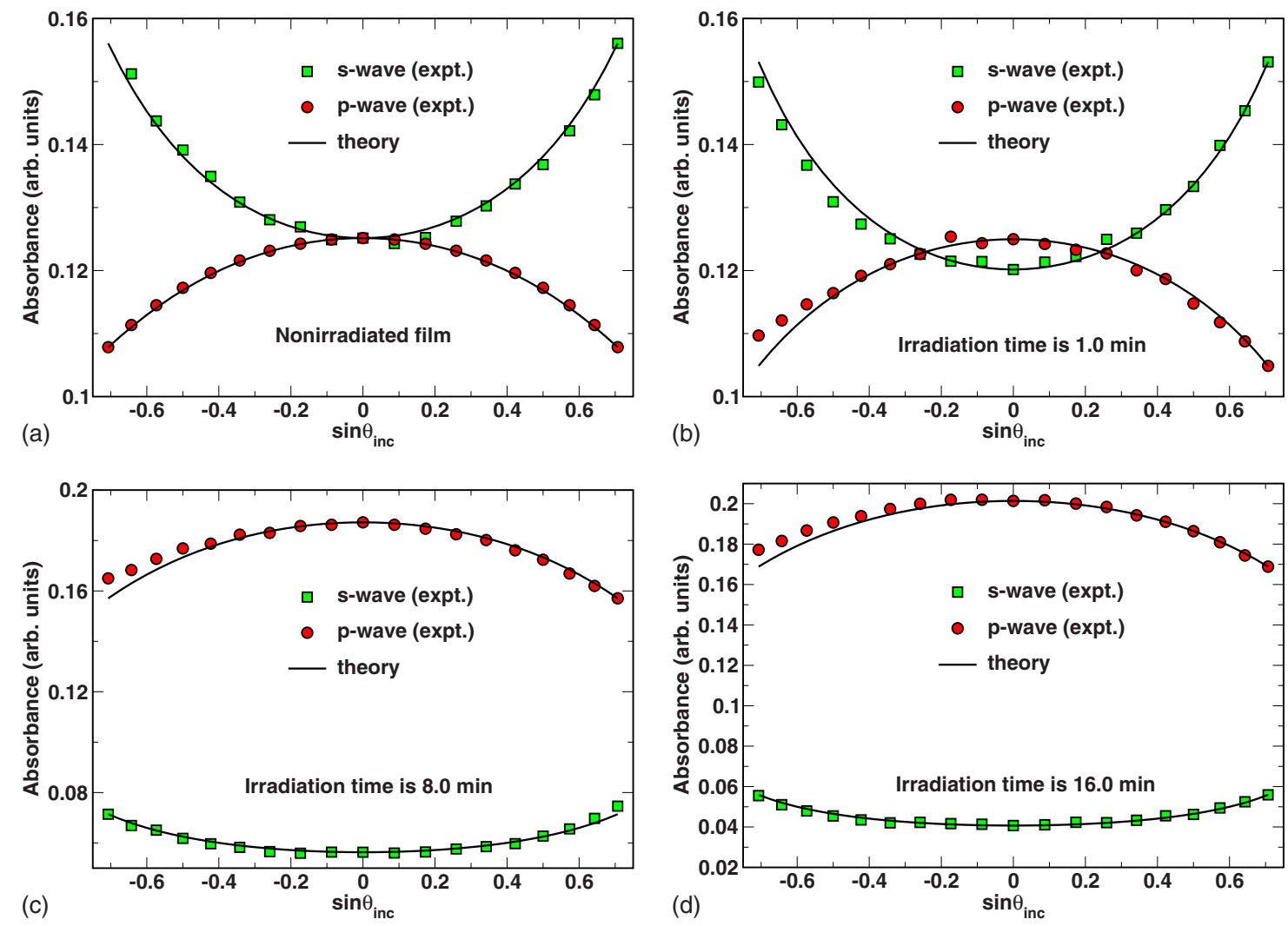

FIG. 8. (Color online) Absorbance as a function of the sine of incidence angle. Circles and squares represent the data measured for testing beam linearly polarized parallel ( $p$-polarized wave) and perpendicular ( $s$-polarized wave) to the plane of incidence, respectively. The theoretical curves are shown as solid lines.

$$
\begin{gathered}
q_{p}=\frac{n_{x}}{n_{z}} \sqrt{n_{z}^{2}-q_{x}^{2}}, \quad q_{s}=\sqrt{n_{y}^{2}-q_{x}^{2}}, \\
\mathbf{V}=\left(\begin{array}{cc}
\mathbf{E} & -\boldsymbol{\sigma}_{3} \mathbf{E} \\
\mathbf{H} & \boldsymbol{\sigma}_{3} \mathbf{H}
\end{array}\right), \quad \mathbf{N}=\frac{2}{\mu} \operatorname{diag}\left(q_{p}, q_{s},-q_{p},-q_{s}\right), \\
\mathbf{E}=\left(\begin{array}{cc}
q_{p} / n_{x} & 0 \\
0 & 1
\end{array}\right), \quad \mathbf{H}=\frac{1}{\mu}\left(\begin{array}{cc}
n_{x} & 0 \\
0 & q_{s}
\end{array}\right) .
\end{gathered}
$$

We can now substitute operator (A17) into the linking matrix (A12) and obtain the transmission and reflection matrices using relations (A13) and (A14). The result is given by

$$
\begin{gathered}
\mathbf{T}=\left(\begin{array}{cc}
t_{p}\left(q_{x}\right) & 0 \\
0 & t_{s}\left(q_{x}\right)
\end{array}\right)=\frac{\mathbf{I}_{2}-\mathbf{P}^{2}}{\mathbf{I}_{2}-\mathbf{U}_{+}^{2} \mathbf{P}^{2}} \mathbf{U}_{+}, \\
\mathbf{R}=\left(\begin{array}{cc}
r_{p}\left(q_{x}\right) & 0 \\
0 & r_{s}\left(q_{x}\right)
\end{array}\right)=\boldsymbol{\sigma}_{3} \frac{\mathbf{I}_{2}-\mathbf{U}_{+}^{2}}{\mathbf{I}_{2}-\mathbf{U}_{+}^{2} \mathbf{P}^{2}} \mathbf{P}, \\
\mathbf{P}=\mathbf{V}_{-} \mathbf{V}_{+}^{-1}, \quad \mathbf{V}_{ \pm}=\left(\begin{array}{cc}
\frac{n_{x}}{\mu n_{m}} q_{m} \pm \frac{n_{m}}{\mu_{m} n_{x}} q_{p} & (\mathrm{~A} 23) \\
0 & \mu^{-1} q_{s} \pm \mu_{m}^{-1} q_{m}
\end{array}\right) .
\end{gathered}
$$

All the matrices that enter Eqs. (A22)-(A24) are diagonal, so that nondiagonal elements of both transmission and reflec- tion matrices vanish. Algebraically, this is a consequence of the diagonal form of the block matrices that define the operator of evolution (A17).

So, absorption of plane waves linearly polarized parallel and perpendicular to the plane of incidence can be characterized by the effective optical densities, $D_{p}$ and $D_{s}$, expressed in terms of the corresponding transmission coefficients,

$$
D_{p, s}\left(\theta_{\text {inc }}\right) \equiv D_{p, s}\left(q_{x}\right)=-2 \ln \left|t_{p, s}\left(q_{x}\right)\right| .
$$

The optical densities [Eq. (A25)] are proportional to the absorbances measured experimentally, $D_{p}^{(\exp )}$ and $D_{s}^{(\exp )}$, and determine the theoretical dependence of the absorbance on the incidence angle, $\theta_{\text {inc }}$ (or, equivalently, on the incidence angle parameter $q_{x}=n_{m} \sin \theta_{\text {inc }}$ ).

In Fig. 8, the experimental data on angular dependence of absorbance measured in the azo-dye SD1 film with the thickness of $15 \mathrm{~nm}$ at different irradiation doses are fitted by the theoretical curves computed from formula (A25).

For our purposes, full description of a rather standard experimental procedure is not important (some details can be found in [42]). So, without going into details we note that the film was illuminated with linearly polarized UV light at varying exposure time by using LED exposure light source. The wavelength and the intensity of the actinic light were $365 \mathrm{~nm}$ and $3.0 \mathrm{~mW} / \mathrm{cm}^{2}$, respectively.

In Fig. 5, the extinction coefficients of the azo-dye layer found as the fitting parameters are plotted as a function of the irradiation time. 
[1] F. Weigert, Verh. Dtsch. Phys. Ges. 21, 479 (1919).

[2] M. Eich, J. H. Wendorff, B. Reck, and H. Ringsdorf, Makromol. Chem., Rapid Commun. 8, 59 (1987).

[3] A. Natansohn, S. Xie, and P. Rochon, Macromolecules 25, 5531 (1992).

[4] Polymers and Other Advanced Materials, edited by P. N. Prasad, J. E. Mark, and T. J. Fai (Plemun Press, New York, 1995).

[5] L. M. Blinov, M. V. Kozlovsky, and G. Cipparrone, Chem. Phys. 245, 473 (1999).

[6] V. G. Chigrinov, Liquid Crystal Devices: Physics and Applications (Artech House, Boston, 1999).

[7] W. M. Gibbons, P. J. Shannon, S.-T. Sun, and B. J. Swetlin, Nature (London) 351, 49 (1991).

[8] M. Schadt, K. Schmitt, V. Kozinkov, and V. Chigrinov, Jpn. J. Appl. Phys., Part 1 31, 2155 (1992).

[9] A. Dyadyusha, T. Marusii, Y. Reznikov, V. Reshetnyak, and A. Khizhnyak, JETP Lett. 56, 17 (1992).

[10] M. O’Neill and S. M. Kelly, J. Phys. D 33, R67 (2000).

[11] V. G. Chigrinov, V. M. Kozenkov, and H.-S. Kwok, Photoalignment of Liquid Crystalline Materials: Physics and Applications, Series in Display Technology (Wiley, Chichester, 2008).

[12] V. G. Chigrinov, V. M. Kozenkov, and H. S. Kwok, in Optical Applications in Photoaligning, edited by L. Vicari (Institute of Physics, Bristol, 2003), pp. 201-244.

[13] B. S. Neporent and O. V. Stolbova, Opt. Spektrosk. 14, 624 (1963).

[14] T. Todorov, N. Tomova, and L. Nikolova, Appl. Opt. 23, 4309 (1984).

[15] Z. Sekkat and M. Dumont, Synth. Met. 54, 373 (1993).

[16] M. Dumont, in Photoactive Organic Materials, edited by F. Kajzar et al. (Kluwer Academic, The Netherlands, 1996), pp. 501-511.

[17] Z. Sekkat, D. Yasumatsu, and S. Kawata, J. Phys. Chem. B 106, 12407 (2002).

[18] R. Raschellà, I.-G. Marino, C. Razzetti, D. Bersani, and P. P. Lottici, J. Opt. Soc. Am. B 24, 504 (2007).

[19] N. C. R. Holme, P. S. Ramanujam, and S. Hvilsted, Appl. Opt. 35, 4622 (1996).

[20] A. Petry, S. Kummer, H. Anneser, F. Feiner, and C. Bräuchle, Ber. Bunsenges. Phys. Chem. 97, 1281 (1993).

[21] U. Wiesner, N. Reynolds, C. Boeffel, and H. W. Spiess, Liq. Cryst. 11, 251 (1992).

[22] L. Blinov, M. Kozlovsky, M. Ozaki, K. Skarp, and K. Yoshino, J. Appl. Phys. 84, 3860 (1998).

[23] O. Yaroshchuk, A. D. Kiselev, Y. Zakrevskyy, J. Stumpe, and J. Lindau, Eur. Phys. J. E 6, 57 (2001).

[24] S. Furumi, M. Nakagawa, S. Morino, and K. Ichimura, Appl. Phys. Lett. 74, 2438 (1999).

[25] H. G. Galabova, D. W. Allender, and J. Chen, Phys. Rev. E 55, 1627 (1997).

[26] S. Perny, P. L. Barny, J. Delaire, T. Buffeteau, C. Sourisseau, I. Dozov, S. Forget, and P. Martinot-Lagarde, Liq. Cryst. 27, 329 (2000).

[27] Y. Wu, J.-I. Mamiya, O. Tsutsumi, A. Kanazawa, T. Shiono, and T. Ikeda, Liq. Cryst. 27, 749 (2000).

[28] H. Akiyama, T. Kawara, H. Takada, H. TakatsuV. Chigrinov, E. Prudnikova, V. Kozenkov, and H. Kwok, Liq. Cryst. 29, 1321 (2002).
[29] V. Chigrinov, A. Muravski, and H. S. Kwok, Phys. Rev. E 68, 061702 (2003).

[30] A. D. Kiselev, V. G. Chigrinov, and D. D. Huang, Phys. Rev. E 72, 061703 (2005).

[31] T. G. Pedersen and P. M. Johansen, Phys. Rev. Lett. 79, 2470 (1997).

[32] T. G. Pedersen, P. M. Johansen, N. C. R. Holme, P. S. Ramanujam, and S. Hvilsted, J. Opt. Soc. Am. B 15, 1120 (1998).

[33] G. A. Puchkovs'ka, V. Y. Reshetnyak, A. G. Tereshchenko, O. V. Yaroshchuk, and J. Lindau, Mol. Cryst. Liq. Cryst. 321, 31 (1998).

[34] S. Sajti, A. Kerekes, M. Barabás, E. Lörincz, S. Hvilsted, and P. S. Ramanujam, Opt. Commun. 194, 435 (2001).

[35] A. D. Kiselev, J. Phys.: Condens. Matter 14, 13417 (2002).

[36] A. Natansohn, P. Rochon, X. Meng, C. Barett, T. Buffeteau, S. Bonenfant, and M. Pezolet, Macromolecules 31, 1155 (1998).

[37] M. Dumont, S. Hosotte, G. Froc, and Z. Sekkat, Proc. SPIE 2042, 2 (1994).

[38] T. Buffeteau and M. Pézolet, Macromolecules 31, 2631 (1998).

[39] A. Kiselev, O. Yaroshchuk, Y. Zakrevskyy, and A. Tereshchenko, Condens. Matter Phys. 4, 67 (2001).

[40] O. V. Yaroshchuk, A. D. Kiselev, Y. Zakrevskyy, T. Bidna, J. Kelly, L.-C. Chien, and J. Lindau, Phys. Rev. E 68, 011803 (2003).

[41] L. S. Kaanumalle, A. Natarayan, and V. Ramamurthy, in Synthetic Organic Chemistry, Molecular and Supramolecular Photochemistry Vol. 12, edited by G. G. Axel and J. Mattay (Dekker, New York, 2005), Chap. 18, pp. 553-618.

[42] A. D. Kiselev, V. G. Chigrinov, H.-S. Kwok, A. Murauski, and A. Muravsky, Proceedings of the Eighth International Display Workshop'08, SID, Nigata, Japan, 2008 (unpublished), pp. 573-574.

[43] V. Chigrinov, S. Pikin, A. Verevochnikov, V. Kozenkov, M. Khazimullin, J. Ho, D. D. Huang, and H. S. Kwok, Phys. Rev. E 69, 061713 (2004).

[44] T. D. Frank, Nonlinear Fokker-Planck Equations, Springer Series in Synergetics (Springer, Berlin, 2005).

[45] C. W. Gardiner, Handbook of Stochastic Methods (SpringerVerlag, Berlin, 1985).

[46] N. G. van Kampen, Stochastic Processes in Physics and Chemistry (North-Holland, Amsterdam, 1984).

[47] H.-P. Breuer and F. Petruccione, The Theory of Open Quantum Systems (Oxford University Press, Oxford, 2002).

[48] M. Dumont and Z. Sekkat, Proc. SPIE 1774, 188 (1993).

[49] P. G. de Gennes and J. Prost, The Physics of Liquid Crystals (Clarendon Press, Oxford, 1993).

[50] M. Born and E. Wolf, Principles of Optics: Electromagnetic Theory of Propagation, Interference and Diffraction of Light, 7th ed. (Cambridge University Press, New York, 1999).

[51] P. M. Chaikin and T. C. Lubensky, Principles of Condensed Matter Physics (Cambridge University Press, Cambridge, 1995).

[52] H. Risken, The Fokker-Planck Equation: Methods of Solution and Applications, Springer Series in Synergetics Vol. 18, 2nd ed. (Springer, Berlin, 1989).

[53] L. C. Biedenharn and J. D. Louck, Angular Momentum in Quantum Physics (Addison-Wesley, Reading, MA, 1981).

[54] L. D. Favro, Phys. Rev. 119, 53 (1960).

[55] M. Doi and S. F. Edwards, The Theory of Polymer Dynamics 
(Oxford University Press, New York, 1988).

[56] J. I. Lauritzen and R. Zwanzig, Adv. Mol. Relax. Processes 5, 339 (1973).

[57] W. T. Coffey, Y. P. Kalmykov, E. S. Massawe, and J. T. Waldron, J. Chem. Phys. 99, 4011 (1993).

[58] J.-L. Dejárdin, G. Debiais, and A. Ouadjou, J. Chem. Phys. 98, 8149 (1993).

[59] J.-L. Dejárdin and Y. P. Kalmykov, Phys. Rev. E 61, 1211 (2000).

[60] Y. P. Kalmykov and K. P. Quinn, J. Chem. Phys. 95, 9142 (1991).

[61] B. U. Felderhof, Phys. Rev. E 66, 051503 (2002).

[62] Y. P. Kalmykov, Phys. Rev. E 65, 021101 (2001).

[63] Y. P. Kalmykov and S. V. Titov, J. Chem. Phys. 126, 174903 (2007).

[64] P. L. Nordio and P. Busolin, J. Chem. Phys. 55, 5485 (1971).

[65] R. Tarroni and C. Zannoni, J. Chem. Phys. 95, 4550 (1991).

[66] G. R. Luckhurst and A. Sanson, Mol. Phys. 24, 1297 (1972).

[67] D. Berggren, R. Tarroni, and C. Zannoni, J. Chem. Phys. 99, 6180 (1993).

[68] A. Ferrarini, P. L. Nordio, and G. J. Moro, in The Molecular Physics of Liquid Crystals, edited by G. R. Luckhurst and C. A. Veracini (Kluwer/Academic, Dordrecht, 1994), pp. 41-69.

[69] A. Brognara, P. Pasini, and C. Zannoni, J. Chem. Phys. 112, 4836 (2000).

[70] P. L. Nordio and U. Serge, in The Molecular Physics of Liquid Crystals, edited by G. R. Luckhurst and G. W. Gray (Academic Press, London, 1979), Chap. 18.

[71] G. Moro and P. L. Nordio, Mol. Cryst. Liq. Cryst. 104, 361 (1984).
[72] I. Dozov, N. Kirov, and B. Petroff, Phys. Rev. A 36, 2870 (1987).

[73] M. P. Fontana, B. Rosi, N. Kirov, and I. Dozov, Phys. Rev. A 33, 4132 (1986).

[74] D. S. Zhang, G. W. Wei, D. J. Kouri, and D. K. Hoffman, Phys. Rev. E 56, 1197 (1997).

[75] A. N. Drozdov and M. Morillo, Phys. Rev. E 54, 931 (1996).

[76] A. N. Drozdov and M. Morillo, Phys. Rev. Lett. 77, 3280 (1996).

[77] A. N. Drozdov, Phys. Rev. E 55, 1496 (1997).

[78] Handbook of Mathematical Functions, edited by M. Abramowitz and I. A. Stegun (Dover, New York, 1972).

[79] Y. A. Kuznetsov, Elements of Applied Bifurcation Theory, Applied Mathematical Sciences Vol. 112, 2nd ed. (Springer, New York, 1998).

[80] J. K. Hale and H. Kocak, Dynamics and Bifurcations, Texts in Applied Mathematics Vol. 3 (Springer, New York, 1991).

[81] F. C. Hoppensteadt, Analysis and Simulation of Chaotic Systems, Applied Mathematical Sciences Vol. 94, 2nd ed. (Springer, New York, 2000).

[82] Ellipsometry and Polarized Light, edited by R. M. A. Azzam and N. M. Bashara (North-Holland, Amsterdam, 1977).

[83] Handbook of Ellipsometry, edited by H. G. Tompkins and E. A. Irene (Springer, Berlin, 2005).

[84] V. Devanathan, Angular Momentum Techniques in Quantum Mechanics, Fundamental Theories of Physics Vol. 108 (Kluwer, New York, 2002).

[85] A. D. Kiselev, J. Phys.: Condens. Matter 19, 246102 (2007).

[86] A. D. Kiselev, R. G. Vovk, R. I. Egorov, and V. G. Chigrinov, Phys. Rev. A 78, 033815 (2008). 